\title{
Single-cell transcriptomics of popliteal lymphatic vessels and peripheral veins reveals altered lymphatic muscle and immune cell populations in the TNF-Tg arthritis model
}

\author{
H. Mark Kenney ${ }^{1,2}$, Chia-Lung Wu ${ }^{1,3}$, Alayna E. Loiselle ${ }^{1,2,3}$, Lianping Xing ${ }^{1,2}$, Christopher T. Ritchlin ${ }^{1,4}$ and
} Edward M. Schwarz $1,2,3,4^{*}$ (i)

\begin{abstract}
Background: Lymphatic dysfunction exists in tumor necrosis factor transgenic (TNF-Tg) mice and rheumatoid arthritis (RA) patients. While joint-draining TNF-Tg popliteal lymphatic vessels (PLVs) have deficits in contractility during end-stage arthritis, the nature of lymphatic muscle cells (LMCs) and their TNF-altered transcriptome remain unknown. Thus, we performed single-cell RNA-sequencing (scRNAseq) on TNF-Tg LMCs in PLVs efferent to inflamed joints versus wild-type (WT) controls.

Methods: Single-cell suspensions of PLVs were sorted for smooth muscle cells (SMCs), which was validated by Cspg4-Cre;tdTomato reporter gene expression. Single-cell RNA-seq was performed on a 10x Genomics platform and analyzed using the Seurat R package. Uniform Manifold Approximation and Projections (UMAPs) and Ingenuity Pathway Analysis software were used to assess cell clusters and functional genomics in WT vs. TNF-Tg populations.

Results: Fluorescent imaging of Cspg4-Cre;tdTomato vessels demonstrated dim PLVs and strong reporter gene expression in the adjacent superficial saphenous vein, which was corroborated by flow cytometry of LMCs and vascular smooth muscle cells (VSMCs) from these vessels. Due to their unique morphology, these populations could also be readily detected by scatter analysis of cells from non-fluorescent mice. Bioinformatics analysis of flow sorted WT and TNF-Tg cells identified 20 unique cell clusters that together were 22.4\% LMCs, 15.0\% VSMCs, and 62.6\% non-muscle cells of 8879 total cells. LMCs and M2-macrophages were decreased, while inflammatory monocytes were increased in TNF-Tg lower limb vasculature. SMC populations were defined by Cald1, Tpm1, and Pdgfrb expression and were enriched in myofibroblast-like gene expression. TNF-Tg LMCs exhibited enhanced functional genomics associated with cell death, phagocyte recruitment, and joint inflammation. Among the most prominent TNF-induced genes in SMCs were Mmp3, Cxcl12, and CCl19, and the most downregulated genes were Zbtb16, Galnt15, and Apod.
\end{abstract}

Conclusions: Single-cell RNA-seq can be used to investigate functional genomics of lower limb vasculature in mice. Our findings confirm the inflammatory transcriptome of TNF-Tg vessels and altered gene expression in SMC populations. This study further supports a potential role of mesenchymal stromal cells in inflammatory-erosive arthritis

\footnotetext{
*Correspondence: Edward_Schwarz@URMC.Rochester.edu

${ }^{1}$ Center for Musculoskeletal Research, University of Rochester Medical

Center, 601 Elmwood Ave, Box 665, Rochester, NY 14642, USA

Full list of author information is available at the end of the article
}

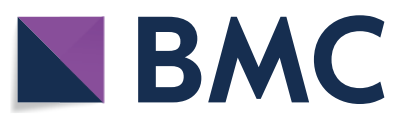

(c) The Author(s) 2022. Open Access This article is licensed under a Creative Commons Attribution 4.0 International License, which permits use, sharing, adaptation, distribution and reproduction in any medium or format, as long as you give appropriate credit to the original author(s) and the source, provide a link to the Creative Commons licence, and indicate if changes were made. The images or other third party material in this article are included in the article's Creative Commons licence, unless indicated otherwise in a credit line to the material. If material is not included in the article's Creative Commons licence and your intended use is not permitted by statutory regulation or exceeds the permitted use, you will need to obtain permission directly from the copyright holder. To view a copy of this licence, visit http://creativecommons.org/licenses/by/4.0/. The Creative Commons Public Domain Dedication waiver (http://creativeco mmons.org/publicdomain/zero/1.0/) applies to the data made available in this article, unless otherwise stated in a credit line to the data. 
pathogenesis, and warrants future studies to define the effects of this TNF-altered transcriptome on PLV function and joint homeostasis.

Keywords: Inflammation, Arthritis, Lymphatics, Vasculature, Smooth Muscle Cells, Fibroblasts, Monocytes, Macrophages, Single-cell RNA-sequencing, Transcriptomics

\section{Background}

While the mechanisms of adaptive immunity in rheumatoid arthritis (RA) have been extensively studied, stromal cells have recently been recognized as an integral component of inflammatory-erosive arthritis pathogenesis $[1,2]$. Specifically, synovial fibroblasts have been demonstrated to mediate a direct role both in the inflammatory $\left(\mathrm{THY} 1^{+}\right)$and erosive $\left(\mathrm{THY}^{-}\right)$processes within the joint [3]. Transcriptomic approaches also identified additional subsets of these synovial fibroblasts, and in particular a peri-vascular fibroblast subset that was selectively increased in RA synovium compared to osteoarthritis [4]. In addition, mechanisms have been proposed relating the recently discovered preinflammatory mesenchymal (PRIME) cells shown to be elevated in peripheral blood preceding an arthritic flare, but then decrease dramatically upon flare onset as the cells presumably migrate into the synovium to differentiate into the pathologic fibroblast subsets [5].

Along with these recent advances implicating a direct relationship between mesenchymal cells in inflammatory arthritis, our previous studies have focused on associated mechanisms related to the pathogenesis of synovial and joint-draining lymphatics in RA [6]. In mice, the synovial lymphatics that drain the ankle joint eventually culminate in collecting popliteal lymphatic vessels (PLVs), two of which traverse on either side of the superficial saphenous vein (SSV) and drain directly into the efferent popliteal lymph node (PLN). The collecting PLVs are largely composed of two cell types, lymphatic endothelial cells (LECs) and lymphatic muscle cells (LMCs). The LMCs exhibit the capacity to both provide vessel tone and pump lymph through coordinated contractions [7]. Previous studies in the tumor necrosis factor transgenic (TNF-Tg) mouse model of RA demonstrated that these PLVs are able to initially drain the inflamed joint effectively leading to dramatic PLN expansion with limited joint disease [8, 9]. However, after a prolonged period, the expanded PLN suddenly collapses with loss of fluid flow, which is concomitant with reduced afferent lymph drainage and PLV contractions correlated with the onset of severe synovitis and bone erosions [10-12]. Ex vivo assessment of cannulated wild-type (WT) vs. TNF-Tg PLV function revealed intrinsic defects in contractility isolated from the afferent joint and efferent PLN regardless of PLN expansion or collapse, suggesting that defects in PLV contractility are the inciting event in this process of lymphatic failure and arthritic progression [13]. Importantly, anti-TNF therapy is able to restore these PLV contractions associated with amelioration of arthritis [11]. We have also translated these findings to human RA, where subjects with active arthritis in their hands demonstrated significantly reduced lymphatic clearance from the web spaces adjacent to the metacarpophalangeal joints by near-infrared imaging of indocyanine green [14].

In our recent work, we have thoroughly evaluated the role of nitric oxide (NO) signaling in mediating the defects in lymphatic contractility noted during the progression of inflammatory arthritis. NO formation through inducible NO synthase (iNOS) produced by peri-lymphatic CD11b ${ }^{+}$myeloid cells [15] and the LECs [16] themselves has been directly implicated to reduce lymphatic contractility with inflammation. In TNF-Tg mice with collapsed PLNs, CD11b ${ }^{+}$and iNOS producing myeloid cells were found to be stagnant within PLVs by intravital microscopy [17] and progressively adhere to the LECs [11], likely involved in the contractile dysfunction. Importantly, treatment with a selective iNOS inhibitor in vivo was sufficient to recover PLV contraction frequency in TNF-Tg mice [16], and TNF-Tg $\mathrm{x}$ iNOS $^{-/-}$mice exhibited reduced joint disease at early time points in females with accelerated arthritic progression $[18,19]$. Detailed assessment of PLV contractility ex vivo revealed that NOS inhibition similarly improved contraction frequency, but was unable to fully recover the reduced contractile amplitude [13]. Together, these studies demonstrated that contractility defects in TNFTg PLVs are only partially related to NO and suggested that the LMCs may be directly damaged and/or transcriptionally modified to explain the persistent PLV dysfunction that remained despite NOS inhibition. Thus, we completed the first single-cell RNA-sequencing (scRNAseq) analysis of PLV-LMCs and the vascular smooth muscle cells (VSMCs) of the adjacent SSV from mice, to define the transcriptome of these cell populations based on functional genomics. We also compared the transcriptomes of the identified LMC and VSMC clusters from WT and TNF-Tg mice to gain insights on the prototypic smooth muscle cell (SMC) genes expressed by these cells, and alterations in this homeostatic transcriptome in the setting of chronic TNF-induced inflammatory-erosive arthritis. 


\section{Methods}

\section{Mouse models and treatments}

Animal experiments were approved by the University Committee for Animal Resources at the University of Rochester. All mice used for this study were maintained on a C57BL/6J genetic background. For the initial experiments to enrich for PLV-LMCs and SSVVSMCs by fluorescence activated cell sorting (FACS) of tdTomato $(\mathrm{tdT})^{+}$cells, male and female Cspg4-Cre mice (Jackson Laboratory \#029926) [20] were crossed with Ai9-tdTomato reporter animals (Jackson Laboratory \#007909) [21], as previously described [22]. Csgp4$\mathrm{Cre}^{+/-}$, Ai9-tdTomato ${ }^{+/-}$offspring $>8$ months old were used for the experiments $(n=3$ mice / experiment, 2 replicates, $n=6$ total mice), while Cspg4-Cre ${ }^{-1-}$, Ai9tdTomato $^{+/-}$littermates $(n=1$ mouse / experiment, $n$ $=2$ total mice) were used as negative controls to accurately gate for $\mathrm{tdT}^{+}$cells. To evaluate PLV-LMCs and SSV-VSMCs by scRNAseq in inflammatory-erosive arthritis, 8-9-month-old TNF-Tg (3647 line, $n=3$ mice) and WT mice ( $n=3$ mice) were used. Only male WT and TNF-Tg mice were used for the study given the early mortality of TNF-Tg females [19]. The TNFTg mice were originally obtained by Dr. George Kollias $[23,24]$ and have been maintained at the University of Rochester.

The experiments to validate the scRNAseq findings by whole mount immunofluorescent microscopy were performed in Pdgfrb-CreER (Jackson Laboratory \#030201) [25], Acta2-Cre (Jackson Laboratory \#029925) [20], and Acta2-CreER (a gift from Dr. Ivo Kaljzic at the University of Connecticut) [26] all crossed into Ai9-tdTomato reporter models. For the CreER experimental mice, tamoxifen (Millipore Sigma Cat\# T5648) was dissolved in corn oil (Millipore Sigma Cat\# C8267) at $10 \mathrm{mg} / \mathrm{mL}$. In the Pdgfrb-CreER mice (2.5 months old), tamoxifen administration was performed by weight at $0.1 \mathrm{mg} / \mathrm{g}$ intraperitoneal, while for the adult Acta2-CreER (5 months old) a standard volume of $200 \mu \mathrm{L}$ was injected intraperitoneal for 5 consecutive days. To confirm efficiency of the intraperitoneal tamoxifen administration, a Pdgfrb-CreER mouse was also induced via local administration of $10 \mu \mathrm{L}$ into the footpad to drain directly into the PLVs for 5 consecutive days. Evaluation of $\mathrm{tdT}^{+}$cells after tamoxifen induction was performed at least 7 days after the final injection.

The following primer sequences were used for genotyping of TNF-Tg and Cre lines:

TNF-Tg Forward: 5'-TAC-CCC-CTC-CTT-CAGACA-CC-3'

TNF-Tg Reverse: 5'-GCC-CTT-CAT-AAT-ATCCCC-CA- $3^{\prime}$
Cre Forward: 5'-CCT-GGA-AAA-TGC-TTC-TGTCCG-TTT-GCC-3'

Cre Reverse: 5'-GAG-TTG-ATA-GCT-GGC-TGGTGG-CAG-ATG-3'

\section{Tissue harvest and dissociation}

Experimental mice were administered a lethal dose of ketamine / xylazine, and the fur on the hindlimbs was removed with depilatory cream. Evan's blue dye (2\%, Millipore Sigma Cat\# E2129) was administered into the hindpaws to be absorbed by the PLVs. Before beginning the dissections, all equipment and gloves were cleaned thoroughly with RNase Zap (Ambion, Cat\# AM9780) and $70 \% \mathrm{EtOH}$. The hindpaw was then secured to a silicone dish, and an incision was made on the posterolateral surface of the calf. The skin was removed to reveal the two PLVs (blue from the dye) that run parallel to the SSV, as previously described [22]. Forceps were used to grasp all three vessels (two PLVs and the SSV), and the vessels were dissected away from the underlying tissue then stored on ice in $200 \mu \mathrm{L}(0.65 \mathrm{~mL}$ Eppendorf tube) of Dulbecco's modified Eagle's medium (DMEM) + GlutaMAX (Gibco, Cat\# 10566-016) supplemented with 10\% fetal bovine serum (FBS; Millipore Sigma Cat\# F0926). After the vessels from all the mice were dissected, the vessels were sequentially placed under a microscope in a drop of the supplemented DMEM and the adipose tissue surrounding the vessels was shred by forceps to mechanically release the vessels from the fat, then the tissue was placed back on ice in the $200 \mu \mathrm{L}$ of media. The bottom and top of a $100 \times 15 \mathrm{~mm}$ petri dish (Falcon Cat\# 351029) were both filled with sterile Dulbecco's phosphate buffered saline (DPBS; Gibco Cat\# 14190-144). The vessels were sequentially washed in the bottom and top of the petri dish, then cut into 4 or more small pieces. The vessels from one mouse (both hindlimbs) were dissociated into single cells together in $1 \mathrm{~mL}$ (1.5 mL Eppendorf tube) of Accumax (Innovative Cell Technologies Cat\# AM-105) rotating for $1 \mathrm{~h}$ at room temperature. A $70 \mu \mathrm{m}$ MACS SmartStrainer (Miltenyi Biotec Cat\# 130098-462) was placed on a 15-mL tube and wet with $3 \mathrm{~mL}$ of $10 \%$ FBS diluted in DPBS. The $1 \mathrm{~mL}$ of Accumax with suspended cells was then passed through the strainer into the $15-\mathrm{mL}$ tube, and $1 \mathrm{~mL}$ of $10 \%$ FBS was used to wash the tube used for the enzymatic digestion then similarly passed through the strainer. This process was performed sequentially for all samples for a given experiment, thus pooling all cells from $n=3$ mice. The cells were then pelleted at $300 \mathrm{~g}$ and $4{ }^{\circ} \mathrm{C}$, and the supernatant was carefully removed until approximately $500 \mu \mathrm{L}$ remained. An additional $500 \mu \mathrm{L}$ of DPBS was then added and the cells were resuspended. The tube was then lightly vortexed to 
rinse the sides of the tube, and the $1 \mathrm{~mL}$ of total fluid was transferred to a $1.5-\mathrm{mL}$ Eppendorf tube. The cells were then pelleted at $600 \mathrm{~g}$ and $4{ }^{\circ} \mathrm{C}$, then resuspended in 500 $\mu \mathrm{L}$ of DPBS. One drop of NucBlue Fixed Cell Stain (DAPI formulation, Thermo Fisher Scientific Cat\# R37606) was added to label dead cells. The cells were then transferred to the Flow Cytometry Core (FCC) at the University of Rochester on ice.

\section{FACS and single-cell library preparation}

The day before the experiment, 1.5-mL Eppendorf tubes for cell collection by FACS were filled with $1.5 \mathrm{~mL}$ of $100 \%$ FBS. Before transfer of the cells to the FCC, the $100 \%$ FBS was removed and replaced with $150 \mu \mathrm{L}$ of $2 \%$ FBS diluted in DPBS. Both an $85 \mu \mathrm{m}(\mathrm{tdT} 1)$ and $100 \mu \mathrm{m}$ (tdT2, WT, and TNF-Tg) nozzle was used for the FACS on a BD FACSArea II instrument sorted with a purity mask. The tdT cell samples from the Cspg4-Cre;Ai9tdTomato mice were sorted using the strategy outlined in Fig. 1 for all cells by forward/side scatter, $\mathrm{DAPI}^{-}$live cells, and then $\operatorname{tdT}^{+}$cells as the final gate for collection. The WT and TNF-Tg cell samples were sorted using the gating strategy described in Fig. 2 specific for high forward/side scatter events. These non-fluorescent samples were similarly sorted for DAPI- live cells as the final gate for collection. Importantly, after the first experiment conducted on tdT1, we modified a number of parameters in order to enhance cell viability post-sorting. The following parameters for experiments tdT2, WT, and TNF-Tg were changed: 1) Increased nozzle size from $85 \mu \mathrm{m}$ to $100 \mu \mathrm{m} ; 2)$ Reduced the possibility of cell adhesion to the FACS collection tube by filling the collection tube with $100 \% \mathrm{FBS}$ held at $4^{\circ} \mathrm{C}$ overnight before replacing with the collection media; and 3) Relied on FACS sorting of DAPI ${ }^{-}$ live cells to quantify viability, and omitted steps to measure viability before sequencing given cell count and time constraints. These parameter modifications may explain the slight difference in events and cell counts among distinct scRNAseq experiments.

The cells were immediately transferred to the Genomics Research Center at the University of Rochester for single-cell library preparation. The sorted cells were counted and then loaded on a Chromium Single-Cell Instrument (10x Genomics, Pleasanton, CA, USA) to generate singlecell Gel Bead-in-Emulsions (GEMs). Library generation for scRNAseq was accomplished using the Chromium Single-Cell 3' Library \& Gel Bead Kit (10x Genomics) based on the manufacturer's instructions. After reverse transcription of the GEMs, the barcoded cDNA was purified with DynaBeads MyOne Silane Beads (Thermo Fisher Scientific Cat\# PN37002D) and amplified by PCR for $3^{\prime}$ cDNA library construction. Illumina's (San Diego, CA, USA) NextSeq 550 (tdT1 sample) and NovaSeq 6000
(tdT2, WT, and TNF-Tg samples) sequencers were then used to generate the sequenced data. Note, when evaluating the relationship between FACS event and sequenced cell counts, many factors such as adhesion of cells to the collection tube, cell loss when concentrating cells for sequencing, and/or variable single-cell capture efficiency may lead to different total downstream sequenced cell numbers. Nevertheless, our FACS and sequencing strategies provide an optimal condition for enhanced cell viability post-sorting and high single-cell capture efficiency.

\section{Single-cell RNA-seq data analysis}

The filtered feature matrices were imported and analyzed using the Seurat packages (Seurat v4.0.3) in RStudio (v1.2.1335; R v4.1.1) [27-30]. A shared nearest neighbor (SNN) clustering algorithm was used to embed the data into 2D space using a Uniform Manifold Approximation and Projection (UMAP) [31]. The data was processed according to the instructions of the Seurat $R$ package and the cell clusters were then defined using resolution $=0.5$. For Fig. 3, the clusters were generated in an unsupervised manner, while generation of cell clusters for Figs. 1 and 2 and Supplementary Figure 7 was minimally supervised for comparable clustering of the SMC populations as in Fig. 3. For example, prior to user supervision for Fig. 1, the VSMC population was split into 2 clusters that were then merged together as determined by unsupervised clustering when more cell types were sequenced in Fig. 3. Multiple scRNAseq datasets were integrated using commonly expressed gene anchors, while the identities of the original datasets could be extracted for comparative analysis. The "FindAllMarkers" command was used to identify the differentially expressed genes among each cluster, while "FindMarkers" with identities specified (i.e., WT or TNF-Tg, LMC or VSMC) was used to determine changes in gene expression between groups. These commands calculate the $\log _{2}$ fold-change $(\log 2 \mathrm{FC})$ and false discovery rate (FDR) based on statistical analysis using the nonparametric Wilcoxon rank sum test between the specified groups. The R package ggplot2 (v3.3.5) [32] was used to create the violin plots for comparing gene expression.

\section{Pathway analysis}

Differentially expressed genes (DEGs) between TNF$\mathrm{Tg}$ and WT LMCs (Supplementary Materials) were uploaded into the Ingenuity Pathway Analysis (IPA; July 2021 release, Qiagen, Hilden, Germany) software. The "Expression" core analysis was performed based on expression log ratio. Within the core analysis, the graphical summary, canonical pathways, and diseases and functions (inflammatory response) panels were directly evaluated. The graphical summary was provided directly from IPA, while the additional gene expression 


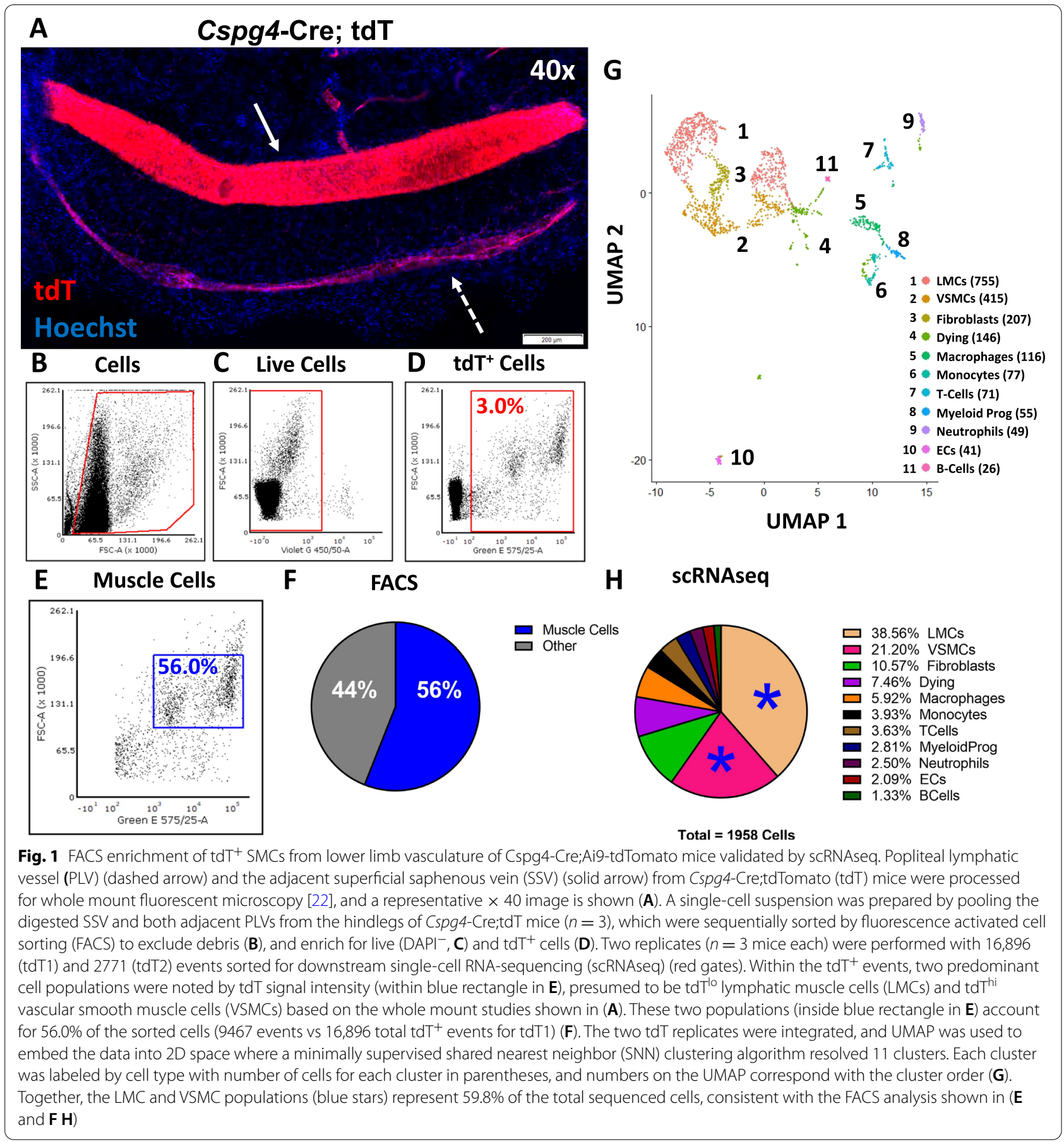

and pathway graphs were generated in GraphPad Prism (v9.1.0, San Diego, CA, USA) based on exported Excel sheets.

\section{Immunostaining and fluorescent microscopy}

PLVs with or without the adjacent SSV were processed for whole mount immunofluorescent microscopy, as previously described [22]. Briefly, after dissection the vessels were fixed in $10 \%$ normalized buffered formalin (NBF) for $30 \mathrm{~min}$, and then washed in $0.1 \%$ Triton X-100 (Millipore Sigma Cat\# X100) / 1× TBS (Bio-Rad Cat\# $1706435)$ solution $(3 \times, 10 \mathrm{~min})$ rocking at room temperature. The vessels were then permeabilized overnight in $0.3 \%$ Triton X-100/ $1 \times$ TBS solution rocking at $4{ }^{\circ} \mathrm{C}$. The 
vessels were blocked in 5\% normal goat serum (Thermo Fisher Scientific Cat\#50062Z) / 0.3\% TritonX-100 / 1× TBS solution rocking at room temperature for $1 \mathrm{~h}$. The vessels were incubated overnight rocking at $4{ }^{\circ} \mathrm{C}$ in primary antibody solution diluted in $5 \%$ normal goat serum / 0.3\% TritonX-100 / 1× TBS; anti-alpha smooth muscle actin ( $\alpha$ SMA) Alexa Fluor 488 conjugated antibody (Thermo Fisher Scientific Cat\# 53-9760-82) was used at 1:100 dilution. To wash the primary antibody, the vessels were incubated with $0.1 \%$ TritonX-100 / $1 \times$ TBS $(3 \times, 10 \mathrm{~min})$ then mounted on a microscope slide with 1 drop of ProLong Gold Antifade Mountant (Thermo Fisher Scientific Cat\# P36930) and NucBlue Live ReadyProbes (Hoechst 33342 formulation; Thermo Fisher Scientific Cat\# R37605). The slides were then imaged by an Olympus VS120 slide scanner or Nikon A1R HD confocal microscope.

\section{Micro-computed tomography $(\mu \mathrm{CT})$}

After vessel dissection, the ankles were harvested and submitted for $\mu \mathrm{CT}$ at the Biomechanics and Multimodal Tissue Imaging Core in the Center for Musculoskeletal Research at the University of Rochester. The ankle joints were imaged using the Scanco Medical VivaCT 40 with the following imaging parameters: $55 \mathrm{kV}, 145 \mu \mathrm{A}, 300 \mathrm{~ms}$ integration time, $2048 \times 2048$ pixels, 1000 projections over $180^{\circ}$, and resolution of $17.5 \mu \mathrm{m}$ isotropic voxels. The DICOM files were then imported into Amira software (v2020.1, Thermo Fisher Scientific, Hillsboro, OR, USA) and the talus was segmented for bone volume measurements, as previously described [33]. Briefly, a set threshold of 2500 Hounsfield units (HU) was used to define bone, and the talus was segmented in the Segmentation Editor. A "Volume Rendering" module with lookup table set to 2500-4500 HU was used for visualization, while "Generate Surface" and "Surface View" modules were used to overlay the talus segmentation in the image. The "Material Statistics" module on the label field defining the talus was used to extract the volume measurements. The volume measurements were exported to GraphPad
Prism for statistical analysis using an unpaired $t$-test to compare WT and TNF-Tg talus bone volumes.

\section{Statistical analysis}

To statistically evaluate changes in gene expression across the scRNAseq experiments, the non-parametric Wilcoxon rank sum test was performed in $\mathrm{R}$ between groups with FDR (adjusted $p$-value) of $<0.01$ considered significant. All other statistical analysis was performed in GraphPad Prism.

\section{Data availability}

The scRNAseq datasets generated in the current study are deposited on NIH Gene Expression Omnibus (GEO; accession number GSE190999), and source code will be provided upon reasonable request. Excel sheets describing the differentially expressed genes for (1) tdT-WT integrated clusters (Fig. 2), (2) WT-TNF integrated clusters (Fig. 3 and Supplementary Figure 2), (3) LMC-VSMC cluster subsets from WT-TNF integration (Supplementary Figure 7), (4) LMC cluster in the WT vs TNF-Tg condition (Fig. 5, Table 2, and Table 3), and (5) VSMC cluster in the WT vs TNF-Tg condition (Table 2 and Table 3) are provided in the Supplementary Materials. All other datasets generated or analyzed during this study are either included in this published article (and its supplementary information files) or will be made available upon reasonable request.

\section{Results \\ FACS enrichment of tdT ${ }^{+}$SMCs from lower limb vasculature of Cspg4-Cre;Ai9-tdTomato mice validated by scRNAseq}

Based on our recent demonstration of tdT reporter gene expression in LMCs and VSMCs of lower limb vasculature from Cspg4-Cre;Ai9-tdT mice [22], we aimed to utilize the reporter gene expression as an approach to isolate primary LMCs and VSMCs for scRNAseq. Fluorescent microscopy of the isolated PLV and adjacent SSV demonstrated that the VSMCs exhibited more intense

(See figure on next page.)

Fig. 2 Strategic gating of unlabeled vasculature derived cells from WT mice enriches for SMCs. Further fluorescence activated cell sorting (FACS) analysis of tdTomato (tdT) sorted cells from Cspg4-Cre;Ai9-tdT mice described in Fig. 1 revealed that selectively gating for high side and forward scatter events (A) enriched for the $\mathrm{tdT}^{+}$smooth muscle cell (SMC) populations, where the percent of $\mathrm{tdT}^{+}$cells relative to total gated live cells increased from 3.0\% (gating as in Fig. 1) to $60.0 \%$ (B). Moreover, 83.2\% of these tdT ${ }^{+}$events represent the SMC populations (relative to $56.0 \%$ as in Fig. 1) (C). Popliteal lymphatic vessels (PLVs) and superficial saphenous veins (SSVs) from 8-9-month-old wild-type (WT) mice $(n=3)$ were harvested, and the gating strategy represented in $\mathbf{A}$ was performed and $\mathrm{DAPI}^{-}$live events were selected (13,130 sorted events) for downstream single-cell RNA-sequencing (scRNAseq). The tdT and WT datasets were integrated and a total of 7446 cells (1958 tdT and 5488 WT) were analyzed by minimally supervised shared nearest neighbor (SNN) clustering algorithms in Seurat. Each cluster was labeled by cell type and the total number of cells for each cluster is shown in parentheses (D). A heatmap represents the top 3 differentially expressed genes for all 18 cell clusters (duplicate genes omitted) with each cluster color coated and in the same cluster order (left to right) as the UMAP in (D) (E). A full gene list for each cluster is provided in the Supplementary Materials. Overlay of the two tdT replicates with the WT sample demonstrated remarkable conservation of cell types with limited batch effect (F). UMAPs of the tdT replicates and WT sample are shown side-by-side to further depict the conservation of cell types, and proportional changes in cell populations with the two different sorting strategies for tdT and WT samples (G) 


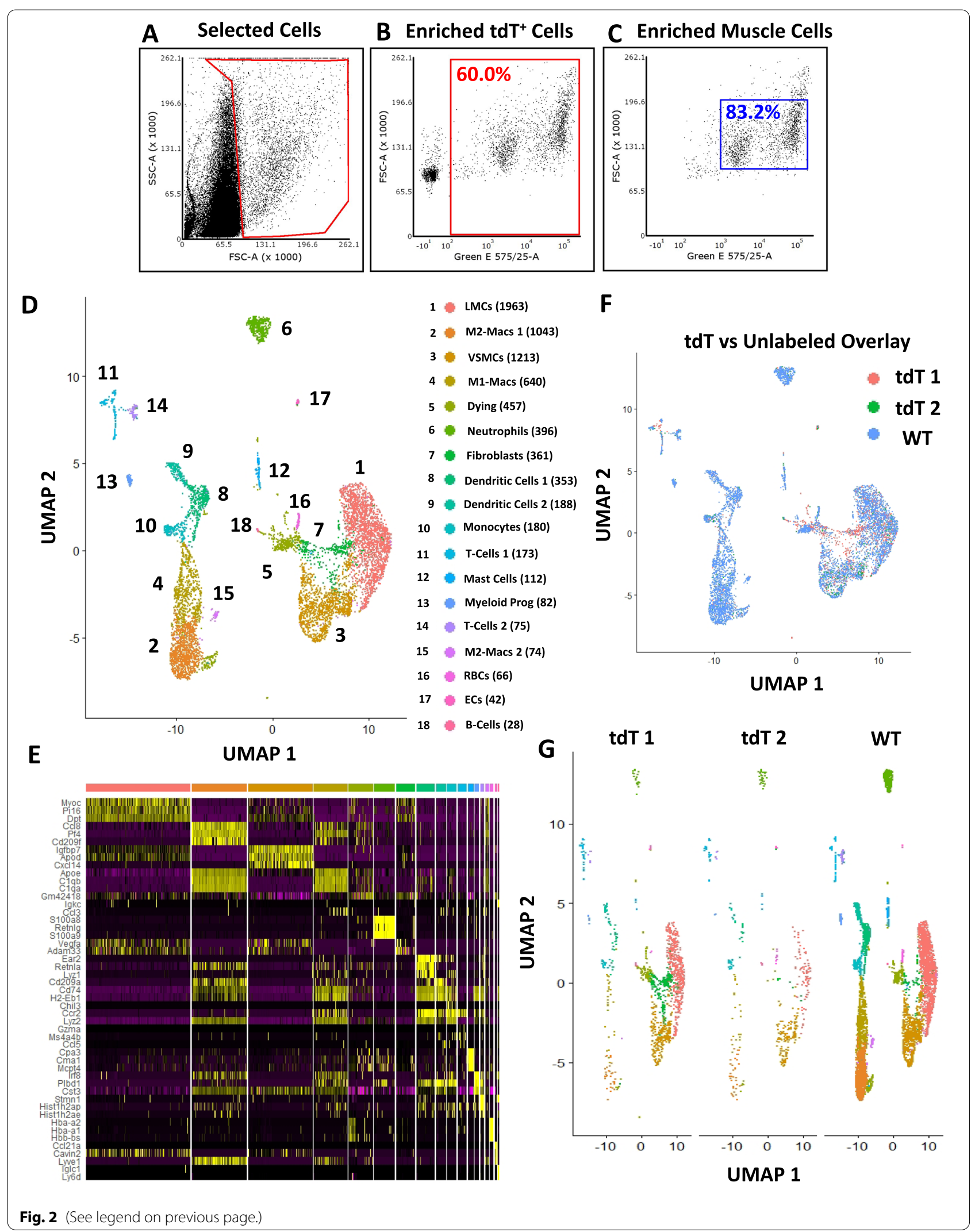


fluorescence than LMCs (Fig. 1A). Quantification of reporter tdT signal intensity in these vessels confirmed that SSVs were significantly brighter $(63.83 \pm 5.43$ artificial units (AU)) than PLVs $(22.28 \pm 5.45 \mathrm{AU})(p<0.05$ by unpaired $t$-test, $n=5$ vessels each group). To assess the utility of this fluorescent label for isolation of the SMC populations, we pooled single-cell suspensions from digested PLVs and associated SSVs from Cspg4Cre;Ai9-tdT mice for FACS enrichment of $\mathrm{tdT}^{+}$cells (Fig. 1B-D). Post-sort analysis of the $\mathrm{td}^{+}$cells revealed two distinct populations based on $\mathrm{td} \mathrm{T}$ expression, which accounted for $56.0 \%$ of the sorted cells isolated for scRNAseq (Fig. 1E, F). For validation of internal consistency with this FACS protocol, the tdT sort was performed twice with each replicate referred to as tdT1 and tdT2. The scRNAseq of both tdT replicates was performed separately, and then merged together for analysis on an integrated dataset. After removing low-quality cells, a total of 1958 cells were used for downstream analysis. With a minimally supervised clustering approach (defined in "Methods"), we identified 11 cell populations (Fig. 1G), 3 of which were determined to be stromal cells due to high expression of Prrx1; hematopoietic cells were identified by Ptprc (CD45) enrichment, while remaining low-quality, dying cells were defined by enhanced mitochondrial gene expression (i.e., $m t$-Cytb) (Supp. Fig. 1). Remarkably, the clusters identified as the SMC populations made up $59.8 \%$ of the cells analyzed by scRNAseq (Fig. $1 \mathrm{H}$ ), similar to the proportion predicted by FACS in Fig. 1F.

\section{Strategic gating of unlabeled vasculature derived cells from WT mice enriches for SMCs}

A serendipitous finding of our initial scRNAseq experiment with Cspg4-Cre;Ai9-tdT mice was that the reporter $\operatorname{dim}($ LMC) and bright (VSMC) populations identified by tdT fluorescent signal (Fig. 1D) could also be identified by forward and side scatter signals by flow cytometry. Thus, we performed a post hoc analysis to determine if selecting for high forward and/or high side scatter events outside of the predominant cell conglomerate
(Fig. 2A) enriches the $\mathrm{tdT}^{+}$cell population. The results confirmed that the majority $(60.0 \%$ of total events in red gate) are the $\operatorname{tdT}^{+}$cells we aimed to sort for scRNAseq (Fig. 2B). Furthermore, by selecting for high forward and/ or side scatter events, many of the proposed non-SMC events that contaminated the $\mathrm{tdT}^{+}$cells from Fig. 1 were excluded with this approach, increasing the SMC proportion from $56.0 \%$ (Fig. 1F) to $83.2 \%$ (Fig. 2C) of the $\mathrm{tdT}^{+}$ cells sorted in Fig. 1. Based on this analysis, single-cell suspensions from the PLVs and SSVs of 8-9-month-old WT mice $(n=3)$ were sorted by FACS solely based on these high forward and/or side scatter parameters, and scRNAseq was performed on the sorted cells. For scRNAseq analyses, the 7446 total cells from the two tdT replicates and the WT sample were integrated to evaluate conservation of the cell types (Fig. 2D), which identified 18 unique cell clusters defined by their differential gene expression (Fig. 2E). When the datasets were overlayed (Fig. 2F) and shown side-by-side (Fig. 2G), there was remarkable consistency of the cell types between the samples. As further demonstration of the selectivity for tdT expression in the stromal cells, there was a dramatic increase in hematopoietic cell populations (predominately monocytes, macrophages, and neutrophils) for the WT sample, which were relatively sparse in the $\mathrm{tdT}$ replicates. However, with the gating strategy shown in Fig. 2A, the SMCs remained a predominant cell population. In fact, the selectivity of SMCs for high forward (large) and/or high side (irregular shape) scatter events is intuitive based on the known large and elongated cell shape of SMCs.

\section{Decreased LMCs and M2-macrophages with increased inflammatory monocytes from joint-draining vasculature of TNF-Tg arthritic mice}

Based on the success of the sorting strategy introduced in Fig. 2, we repeated this process for 8-9-month-old TNF-Tg mice $(n=3)$. Integration of PLVs and SSVs from WT and TNF-Tg mice identified 20 unique cell clusters from a total of 8879 cells (5488 WT and 3391 TNF-Tg)

\footnotetext{
(See figure on next page.)

Fig. 3 Decreased LMCs and M2-macrophages with increased inflammatory monocytes from peripheral vasculature of TNF-Tg arthritic mice. To compare with the wild-type (WT) dataset described in Fig. 2, popliteal lymphatic vessels (PLVs) and superficial saphenous veins (SSVs) from 8-9-month-old tumor necrosis factor transgenic (TNF-Tg) mice $(n=3)$ with 6963 events were isolated by fluorescence activated cell sorting (FACS) for single-cell RNA-sequencing (scRNAseq). The WT and TNF-Tg datasets were integrated and the unsupervised shared nearest neighbor (SNN) clustering algorithm in Seurat resolved 20 distinct cell clusters from the 8879 total cells (5488 WT and 3391 TNF) analyzed. Each cluster was labeled by cell type with total number of cells for each cluster in parentheses and each cluster numbered on the UMAP (A). Sub-analysis of the top 6 most abundant cell populations in the WT (B) and TNF-Tg $(\mathbf{C})$ samples was performed to assess changes in cell proportions between the conditions. In the 4227 subclustered cells from the WT dataset, a small proportion of the cells represented inflammatory monocytes (1.6\%), while lymphatic muscle cells (LMCs) (33.9\%) and M2 macrophages (26.3\%) were the predominant cell populations (D). In the 2454 subclustered cells from TNF-Tg mice, the inflammatory monocyte population expanded dramatically to $20.1 \%$, while both LMCs and M2 macrophages decreased to $22.5 \%$ and $11.0 \%$, respectively (E). The cell counts and percentages for each cluster between WT and TNF-Tg vasculature are provided in Table 1. Supplementary Figure 4 describes the markers used to define the specific monocyte and macrophage populations, while Fig. 4 and Supplementary Figures 5, 6, and 7 together describe the identification of LMCs
} 


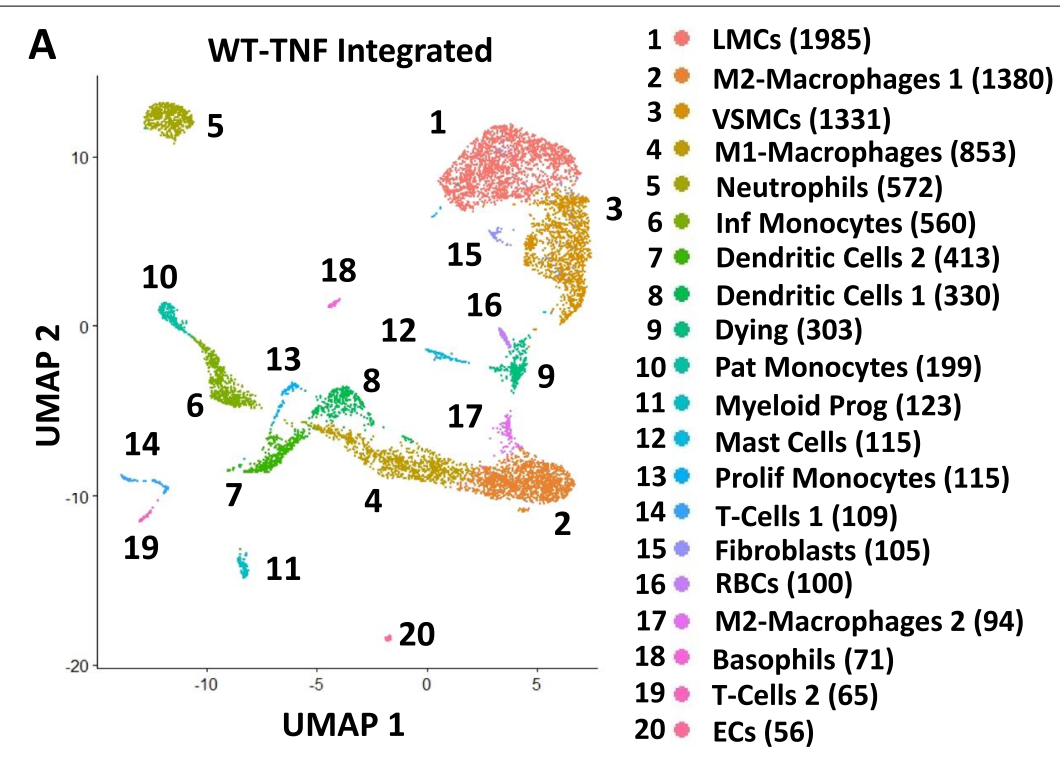

B
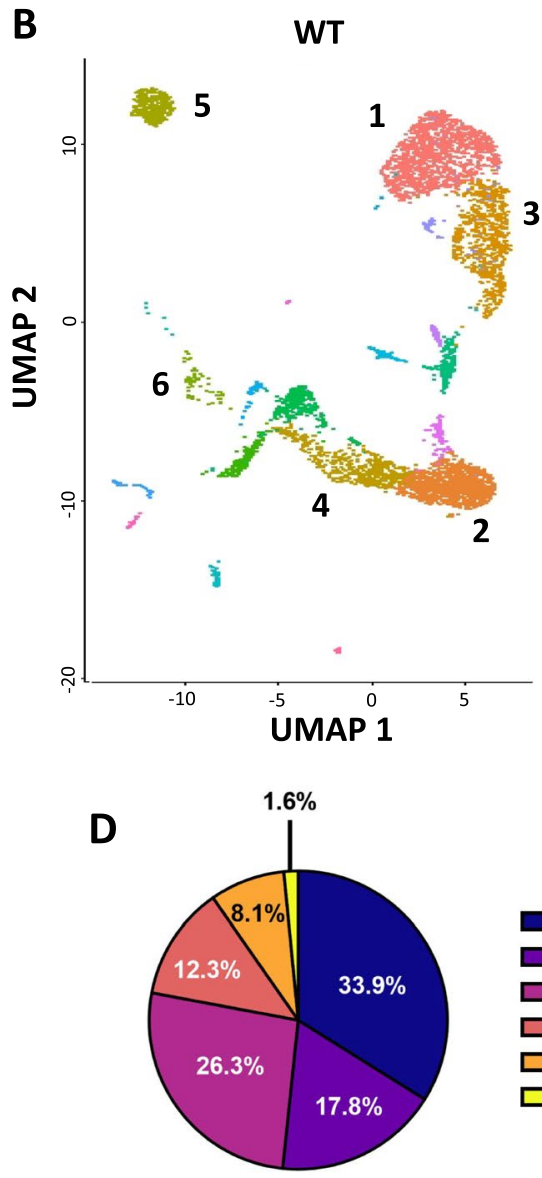

Total $=4227$ Cells
C
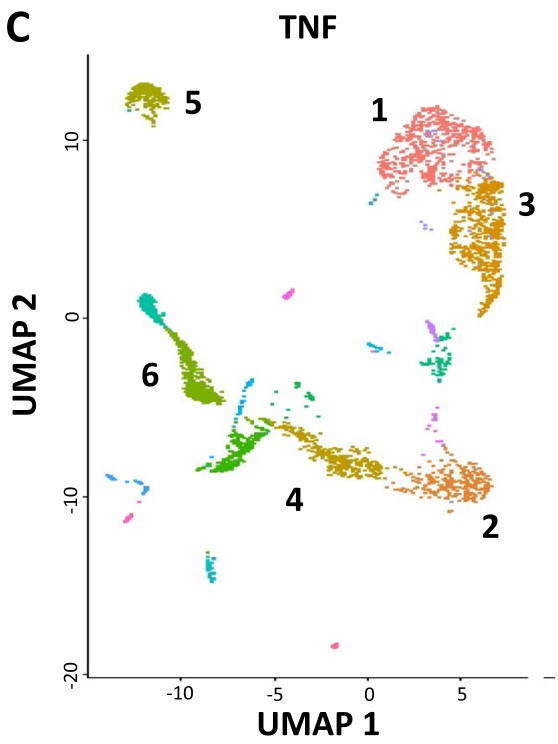

E

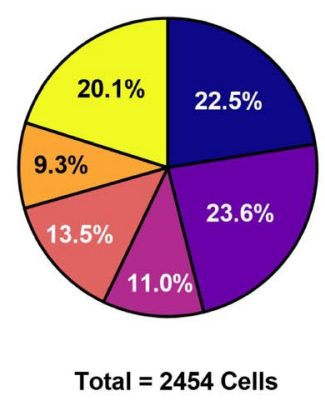

Fig. 3 (See legend on previous page.) 
analyzed (Fig. 3A) with differentially expressed genes for each cluster depicted as a heatmap (Supp. Fig. 2). The 8-9-month-old TNF-Tg mice used in this study were confirmed to have severe arthritis in their ankles afferent to the PLVs by $\mu \mathrm{CT}$ with significantly reduced talus bone volumes (WT $1.39 \pm 0.07$ vs TNF-Tg $0.49 \pm 0.12$ $\mathrm{mm}^{3}, p<0.0001$; Supp. Fig. 3). The various monocyte and macrophage populations were identified as a group based on Itgam (CD11b) expression, and subdivided based on gene enrichment, where patrolling monocytes (Pat Monocytes, cluster 10) express Cx3cr1, inflammatory monocytes (Inf Monocytes, cluster 6) express Ccr2 and $L y 6 c 2$, and the primary M2-macrophage population (M2-Macrophages 1, cluster 2) was defined as $C d 163^{\mathrm{Hi}} / \mathrm{Mrc1}^{\mathrm{Hi}} /$ Lyve $^{\mathrm{Hi}}$ relative to the $\mathrm{Ccr} 2^{+} / \mathrm{Ccl} 2^{\mathrm{Hi}}$ M1-macrophage cluster (cluster 4) [34-37]. Of note was the enrichment of M2-macrophage populations for Lyve 1, previously identified as an essential factor in mediating collagen content in VSMCs [38] (Supp. Fig. 4). The UMAPs of the WT (Fig. 3B) and TNF-Tg (Fig. 3C) cells analyzed by scRNAseq are shown with the top 6 clusters by number of cells identified to evaluate changes in cell proportions between WT and TNF-Tg mice (Fig. 3D, E).
Notably, inflammatory monocytes (cluster 6) expanded dramatically from $1.6 \%$ in WT mice to $20.1 \%$ in TNF$\mathrm{Tg}$ mice. In addition, the LMC population decreased in TNF-Tg mice from $33.9 \%$ in WT to $22.5 \%$ in TNF-Tg, similar to recent reports of a progressive reduction of LMC coverage surrounding synovial lymphatic vessels of TNF-Tg animals [39]. Associated with these changes was a considerable decrease in M2-macrophages from $26.3 \%$ in WT to $11.0 \%$ in TNF-Tg mice, which may negatively impact the homeostasis of LMCs. Changes in all 20 identified cell clusters between WT and TNF-Tg mice are provided in Table 1.

\section{Prototypic SMC markers Cald1, Tpm1, and Pdgfrb detected in LMCs and VSMCs by scRNAseq}

To validate the identification of the SMC clusters, various evaluations of gene enrichment were performed. As expected, the SMC clusters were significantly enriched for Cald1 (Caldesmon; Fig. 4A) [40] and Tpm1 (Tropomyosin alpha-1; Fig. 4B) [41] as typical markers for SMCs [42]. In addition, the SMC and pericyte marker Pdgfrb (Platelet-derived growth factor receptor beta) [43, 44] was significantly expressed in the SMC populations (Fig. 4C).

Table 1 Changes in cell clusters from the peripheral vasculature between WT and TNF-Tg mice by scRNAseq. The cluster numbers and cell types are aligned with those defined in Fig. 3. The cell numbers associated with each cluster are provided for both wild-type (WT) and tumor necrosis factor transgenic (TNF-Tg) groups along with the total cell counts for each cluster when these datasets are integrated together. To examine the changes in cell populations between the WT and TNF-Tg conditions, a percentage for each cell type was calculated by the number of cells in an individual cluster divided by the total cells within a single group (WT or TNF-Tg)

\begin{tabular}{|c|c|c|c|c|c|c|}
\hline Cluster & Cell Type & WT \# & WT \% & TNF \# & TNF \% & Total \# \\
\hline 1 & LMCs & 1433 & 26.11 & 552 & 16.28 & 1985 \\
\hline 2 & M2-Macrophages 1 & 1111 & 20.24 & 269 & 7.93 & 1380 \\
\hline 3 & VSMCs & 751 & 13.68 & 580 & 17.10 & 1331 \\
\hline 4 & M1-Macrophages & 522 & 9.51 & 331 & 9.76 & 853 \\
\hline 5 & Neutrophils & 344 & 6.27 & 228 & 6.72 & 572 \\
\hline 6 & Inf Monocytes & 66 & 1.20 & 494 & 14.57 & 560 \\
\hline 7 & Dendritic Cells 1 & 152 & 2.77 & 261 & 7.70 & 413 \\
\hline 8 & Dendritic Cells 2 & 306 & 5.58 & 24 & 0.71 & 330 \\
\hline 9 & Dying Cells & 236 & 4.30 & 67 & 1.98 & 303 \\
\hline 10 & Pat Monocytes & 7 & 0.13 & 192 & 5.66 & 199 \\
\hline 11 & Myeloid Progenitors & 74 & 1.35 & 49 & 1.45 & 123 \\
\hline 12 & Mast Cells & 98 & 1.79 & 17 & 0.50 & 115 \\
\hline 13 & Prolif Monocytes & 70 & 1.28 & 45 & 1.33 & 115 \\
\hline 14 & T-Cells 1 & 45 & 0.82 & 64 & 1.89 & 109 \\
\hline 15 & Fibroblasts & 75 & 1.37 & 30 & 0.88 & 105 \\
\hline 16 & $\mathrm{RBCs}$ & 54 & 0.98 & 46 & 1.36 & 100 \\
\hline 17 & M2-Macrophages 2 & 80 & 1.46 & 14 & 0.41 & 94 \\
\hline 18 & Basophils & 7 & 0.13 & 64 & 1.89 & 71 \\
\hline 19 & T-Cells 2 & 31 & 0.56 & 34 & 1.00 & 65 \\
\hline \multirow[t]{2}{*}{20} & Endothelial Cells & 26 & 0.47 & 30 & 0.88 & 56 \\
\hline & Total & 5488 & 100 & 3391 & 100 & 8879 \\
\hline
\end{tabular}




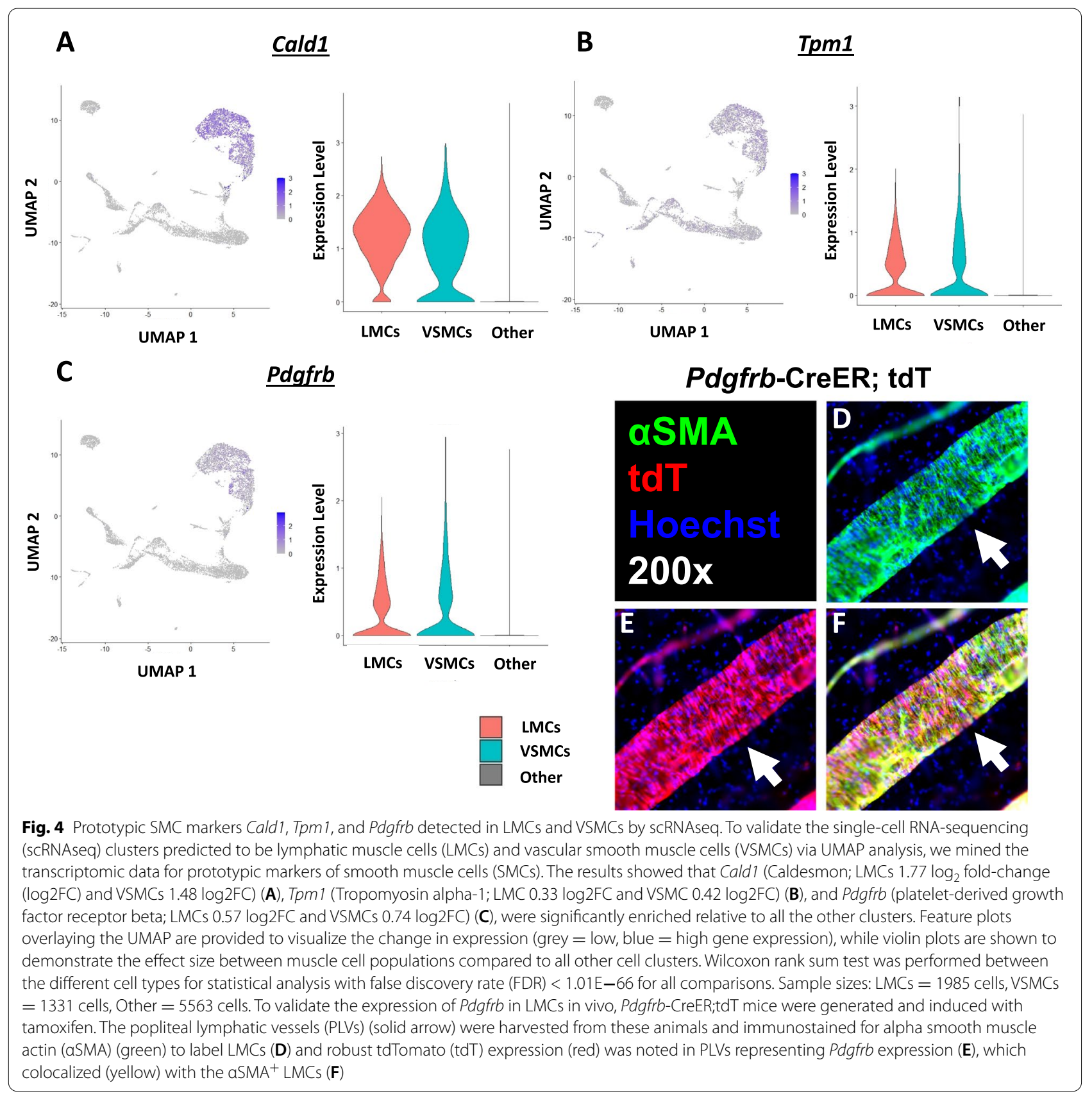

To validate enrichment of $P d g f r b$ expression in the SMCs of these vessels, we generated Pdgfrb-CreER;Ai9-tdT lineage tracing models. After induction with tamoxifen, the SMCs of harvested PLVs and SSVs demonstrated robust tdT expression that colocalized with $\alpha$ SMA (Fig. 4D-F), as expected from the gene enrichment by scRNAseq. Furthermore, the SMC populations were assessed for lymphangiogenic and angiogenic factors, such as Vegfa, Vegfd, and Angpt1 [45, 46], which were all significantly increased in the SMC populations. A combination of muscle-related transcription factors (Prrx1, Twist1, Tead1, Meox2, Id3, and Csrp2), with a role in regulating expression of smooth muscle cell proteins involved in structure and contractility, were also significantly expressed in the SMC clusters. Although none of these transcription factors are specific to SMCs, their combinatorial expression suggests SMC identity (Supp. Fig. 5) [47]. The SMC populations were also directly assessed for common pericyte markers Cspg4, Mcam, and Ngfr [48] all of which were nearly undetectable throughout 
the dataset. Note that the Cspg4-Cre;tdT model used to initially sort SMCs in Figs. 1 and 2 does not presume active expression of Cspg4 in these cells. Indeed, a previous study reported that after early development, limited or absent Cspg4 expression was observed in VSMCs and LMCs using inducible Cspg4-CreER;tdT models [22]. However, non-specific fibroblast genes $D c n, F b \ln 2$, and Pdgfra [49] were highly expressed in the SMC clusters, which suggests that the PLV-LMCs and SSV-VSMCs are myofibroblast-like (Supp. Fig. 6). To differentiate LMCs from VSMCs, known factors produced by VSMCs such as Cst3 (Cystatin $\mathrm{C} / 3 ; 2.60 \log _{2}$ fold-change $(\log 2 \mathrm{FC})$ ) [50], Mgp (Matrix Gla protein; $2.75 \log _{2} \mathrm{FC}$ ) [51, 52], and Bgn (Biglycan; $2.11 \log _{2} \mathrm{FC}$ ) [53, 54] were significantly increased in VSMCs compared to LMCs. Moreover, expression of Ackr3 (Atypical chemokine receptor 3, formerly CXCR7; $1.39 \log _{2}$ FC), known to be selective by $A c k r 3^{\mathrm{GFP}}$ nuclear reporter expression for lymphatic compared to blood vessels in a study specifically investigating the role and noted expression of Ackr3 in LECs [55], was significantly increased in the LMC cluster $(p<2.53 \mathrm{E}-227$ by Wilcoxon rank sum test for all comparisons) (Supp. Fig. 7). Compared to the VSMCs, Vegfd was also significantly increased in the LMC cluster and VEGFD is known to interact specifically with VEGFR3 in mice to promote lymphangiogenesis [56, 57]. However, expression of Vegfc was nearly undetectable throughout the dataset (Supp. Fig. 5).

However, despite expression of these prototypic markers in the SMC clusters, some conventionally muscle-associated genes were not detected in these SMC populations. For example, canonical markers such as Acta2 ( $\alpha \mathrm{SMA}$ ), Myh11 (myosin heavy-chain 11), and Tagln (transgelin) [58] showed limited expression throughout the dataset. Given this unexpected result, we generated two different Acta 2 reporter models, Acta2-Cre;Ai9-tdT and Acta2-CreER;Ai9-tdT mice. Interestingly, tdT expression denoting Acta2 transcription in vivo was also unexpectedly low in PLV-LMCs and SSV-VSMCs in both of these reporter lines, despite robust protein expression of aSMA (Supp. Fig. 8). In addition, the Acta2-CreER;Ai9-tdT mice are reported to exhibit endogenous Cre activation in cells with high aSMA expression, such as the VSMCs of the aorta [26]. However, even after formal induction with intraperitoneal tamoxifen, the tdT expression was limited in the peripheral PLV-LMCs and SSV-VSMCs, suggesting lower Acta2 expression than VSMCs surrounding large arteries that exhibit endogenous tdT expression even without tamoxifen induction. Taken together, we found the SMC populations to be best identified by expression of various myofibroblast genes, SMC-related transcription factors, and lymphangiogenic or angiogenic factors, rather than prototypic SMC genes.

\section{TNF-Tg LMCs exhibit gene expression changes associated with known pathways of joint inflammation and arthritis} To further evaluate changes in PLV-LMC gene expression in TNF-Tg compared to WT mice, we further investigated LMCs (cluster 1 in Fig. 3) between the conditions. $\log _{2} \mathrm{FC}$ was plotted against the FDR to generate a volcano plot showing the most significantly upregulated and downregulated genes where the top 5 genes are annotated for each direction (Fig. 5A). To further highlight the top upregulated and downregulated genes in TNF-Tg SMCs, those genes modified in both LMCs and VSMCs are provided in Table 2, while those specific to LMCs are shown in Table 3. Notably, Edn1 (Endothelin 1), involved in maintenance of vascular tone and inhibiting SMC apoptosis [59], was dramatically downregulated in TNF-Tg LMCs. Zbtb16, a transcription factor previously shown to be downregulated in osteoarthritic cartilage [60], decreased to nearly undetectable levels in TNF-Tg LMCs (WT $85.6 \%$ vs TNF-Tg $21.9 \%$ of cells expressed the gene with $-1.18 \log _{2}$ FC). Related to established changes in synovial fibroblast-like populations in inflammatory arthritis, Thy1 (CD90) demonstrated a significant decrease specifically in TNF-Tg LMCs (Table 3). Interestingly, this finding is consistent with the observation that THY1 ${ }^{-}$fibroblasts are known to be directly involved in

\footnotetext{
(See figure on next page.)

Fig. 5 TNF-Tg LMCs exhibit gene expression changes associated with known pathways of joint inflammation and arthritis. Differentially expressed genes of the lymphatic muscle cell (LMC) cluster (cluster 1 in Fig. 3) from wild-type (WT) vs tumor necrosis factor transgenic (TNF-Tg) mice are shown as a volcano plot (A). Each dot represents a single gene, and black dots showed no significant change from WT (false discovery rate (FDR) $>0.01$, below grey line). The top 5 downregulated (fold-change $<0$ ) and top 5 upregulated (fold-change $>0$ ) in TNF-Tg LMCs are identified by blue dots with gene identifiers. Ingenuity pathway analysis (IPA) was performed on all genes represented in (A), and notably the gene pathway modifications (blue $=$ decreased, orange $=$ increased pathway score) ultimately centered on an increase in "Organismal death" (B). The top 10 most significantly modified canonical pathways (C) and associated inflammatory diseases (D) for TNF-Tg vs WT LMCs were identified, where trends of increased (red), decreased (blue), and unchanged (grey) activity patterns are shown. Specific gene expression patterns in TNF-Tg LMCs were identified for their significant involvement in the "Inflammation of joint" pathways, and representative genes most differentially expressed only in the LMC and vascular smooth muscle cell (VSMC) clusters are indicated: Mmp3 (LMCs 1.65, VSMCs $1.41 \log _{2}$ fold-change (log2FC) in E), CxCl12 (LMCs 1.54, VSMCs $0.69 \log 2 \mathrm{FC}$ in $\mathbf{F}$ ), and CCl19 (LMCs 1.24, VSMCs $2.12 \log 2 \mathrm{FC}$ in $\mathbf{G}$ ). Wilcoxon rank sum test was performed between the same cell types of WT and TNF datasets with an FDR < 4.77E-21 for all comparisons. Sample sizes: WT LMCs $=1433$ cells, TNF LMCs $=552$ cells, WT VSMCs $=751$ cells, TNF VSMCs $=580$ cells
} 


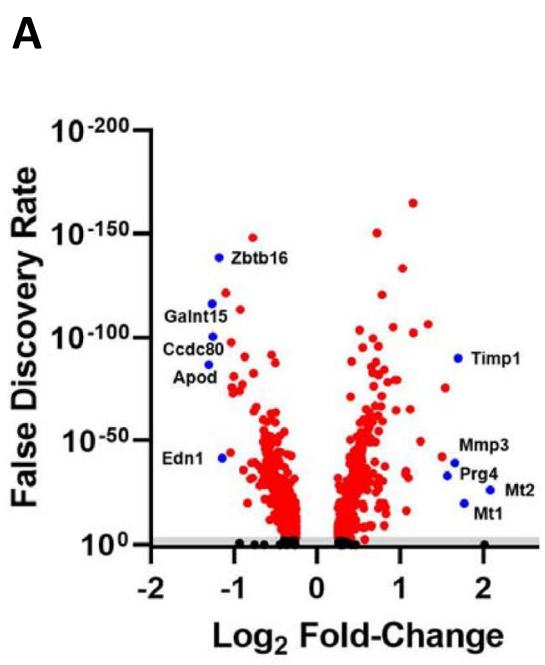

C

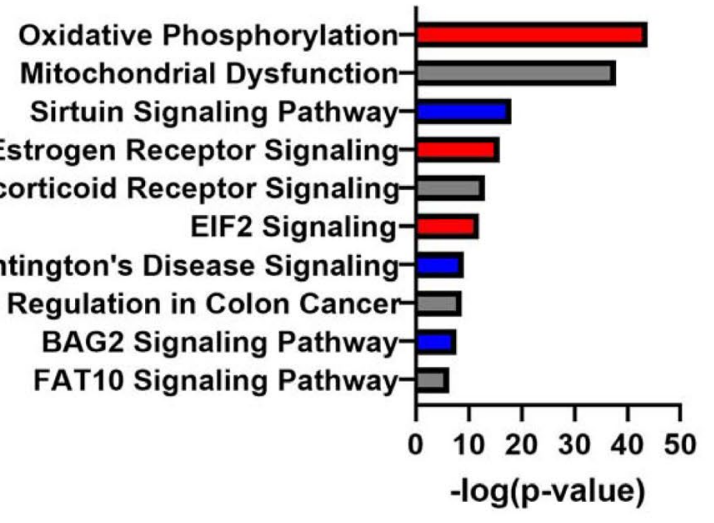

D

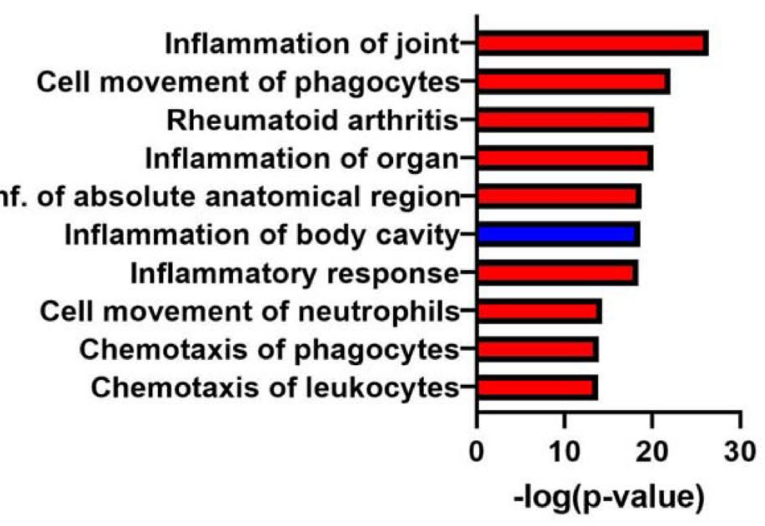

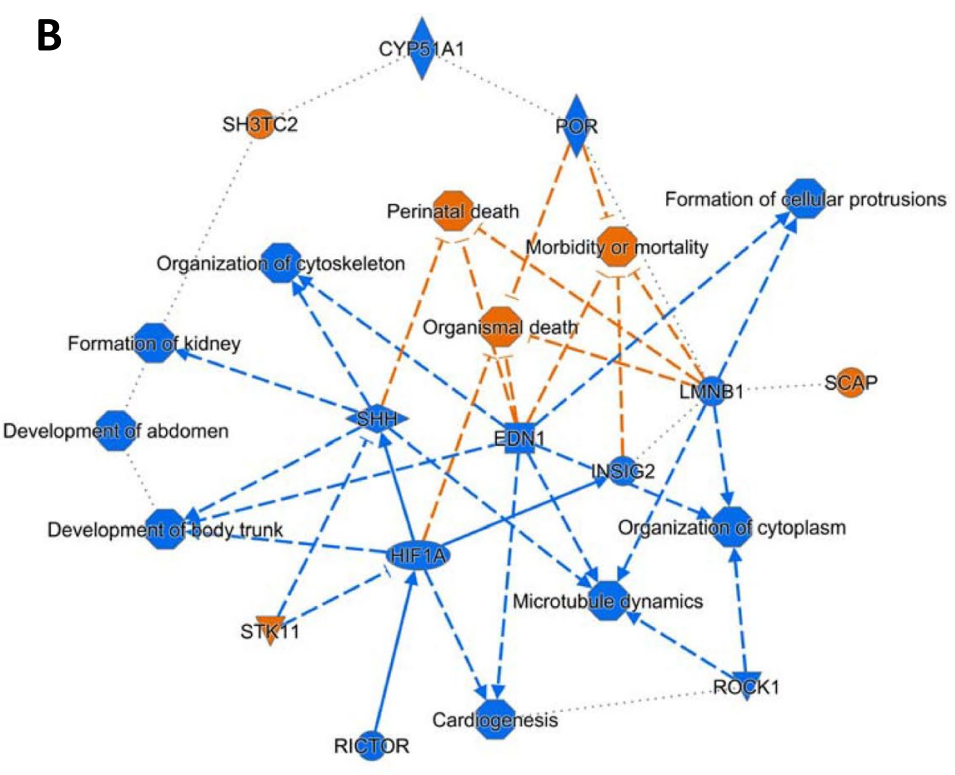

E

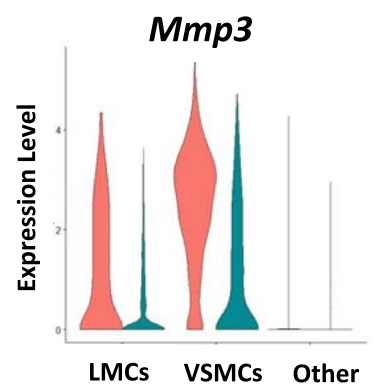

$\mathbf{F}$

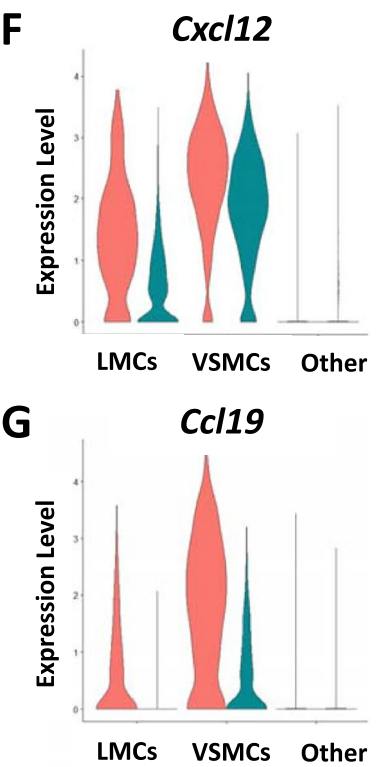

Fig. 5 (See legend on previous page.) 
Table 2 Differentially regulated genes in TNF-Tg vs WT mice common between LMCs and VSMCs. Mt2 (and Mt1, omitted for redundancy), Timp1, Mmp3, Prg4, and Cxcl12 are the top 5 upregulated genes in tumor necrosis factor transgenic (TNF-Tg) lymphatic muscle cells (LMCs) that similarly demonstrate increased expression in TNF-Tg vascular smooth muscle cells (VSMCs) (bold). On the other hand, Apod, Galnt15, Ccdc80, Zbtb16, and KIf9 are the top 5 downregulated genes in TNF-Tg LMCs that were also differentially expressed in TNF-Tg VSMCs (not bold). Proteins coded by the gene, $\log _{2}$ fold-change (log2FC), and false discovery rates (FDRs) for LMCs and VSMCs are provided. The top 5 genes were ranked based on log2FC effect size with an FDR $<0.01$. The genes were selected by evaluating the top up- or downregulated genes for LMCs and listed if the particular genes were also differentially regulated in VSMCs. The LMC and VSMC populations were defined based on the wild-type (WT) and TNF-Tg integrated datasets in Fig. 3. A complete list of differentially expressed genes in TNF-Tg mice for both LMC and VSMC populations is provided in the Supplementary Materials

\begin{tabular}{|c|c|c|c|}
\hline Gene & Protein & Avg $\log _{2} \mathrm{FC}(\mathrm{LMC} \& \mathrm{VSMC})$ & FDR (LMC \& VSMC) \\
\hline Mt2 & Metallothionein 2 & $2.08 \& 1.19$ & $2.26 \mathrm{E}-20 \& 1.04 \mathrm{E}-13$ \\
\hline Timp1 & Timp1 & $1.69 \& 1.61$ & $7.79 \mathrm{E}-91 \& 1.59 \mathrm{E}-65$ \\
\hline Mmp3 & Matrix Metalloproteinase-3 & $1.65 \& 1.41$ & $5.58 \mathrm{E}-40 \& 1.27 \mathrm{E}-61$ \\
\hline $\operatorname{Prg} 4$ & Proteoglycan 4 / Lubrican & $1.56 \& 1.46$ & $7.71 \mathrm{E}-34 \& 1.08 \mathrm{E}-43$ \\
\hline Cxcl12 & C-X-C Motif Chemokine 12 & $1.54 \& 0.69$ & $2.59 \mathrm{E}-76 \& 4.77 \mathrm{E}-21$ \\
\hline Apod & Apolipoprotein D & $-1.30 \&-1.61$ & $1.21 \mathrm{E}-87 \& 3.99 \mathrm{E}-87$ \\
\hline Galnt15 & Polypeptide N-acetylgalactosaminyl-transferase 15 & $-1.26 \&-1.28$ & $5.5 \mathrm{E}-117 \& 4.11 \mathrm{E}-62$ \\
\hline Ccdc80 & Coiled-coil Domain-Containing Protein 80 & $-1.25 \&-1.69$ & $3.8 \mathrm{E}-101 \& 1.95 \mathrm{E}-82$ \\
\hline Zbtb16 & Zinc Finger and BTB Domain-Containing Protein 16 & $-1.17 \&-1.07$ & $2.9 \mathrm{E}-139 \& 1.84 \mathrm{E}-75$ \\
\hline KIfg & Krueppel-like Factor 9 & $-1.10 \&-1.08$ & $2.8 \mathrm{E}-122 \& 1.62 \mathrm{E}-57$ \\
\hline
\end{tabular}

Table 3 Differentially regulated genes in TNF-Tg vs WT mice specific to LMCs. Lbp, Mcoln2, SIc16a2, Adamts1, and Calr are the top 5 upregulated genes that are specific to tumor necrosis factor transgenic (TNF-Tg) lymphatic muscle cells (LMCs) without notable differential gene expression in TNF-Tg vascular smooth muscle cells (VSMCs) (bold). Similarly, Edn1, Thy1, Fbn 1, Cyp2f2, and Clic5 are the top 5 genes specifically downregulated in TNF-Tg LMCs (not bold). Proteins coded by the gene, $\log _{2}$ fold-change (log2FC), and false discovery rates (FDRs) are provided. The top 5 genes were ranked based on log2FC effect size with an FDR $<0.01$. The genes were selected by evaluating the top up- or downregulated genes for LMCs and listed if the particular genes were unique to LMCs and not also differentially regulated in VSMCs. The LMC and VSMC populations were defined based on the wild-type (WT) and TNF-Tg integrated datasets in Fig. 3

\begin{tabular}{lllr}
\hline Gene & Protein & Avg Log $_{2}$ FC & FDR \\
\hline Lbp & Lipopolysaccharide-Binding Protein & $\mathbf{0 . 7 9}$ & $\mathbf{3 . 0 5 E - 3 1}$ \\
Mcoln2 & Mucolipin-2 & $\mathbf{0 . 4 7}$ & $\mathbf{1 . 0 3 E}-\mathbf{4 6}$ \\
Slc16a2 & Monocarboxylate Transporter 8 & $\mathbf{0 . 4 6}$ & $\mathbf{1 . 1 4 E - 3 0}$ \\
Adamts1 & A Disintegrin and Metalloproteinase with Thrombospondin Motifs 1 & $\mathbf{0 . 4 6}$ & $\mathbf{3 . 8 1 E}-\mathbf{0 8}$ \\
Calr & Calreticulin & $\mathbf{0 . 4 5}$ & $\mathbf{6 . 9 7 E - 2 2}$ \\
Edn1 & Endothelin-1 & $\mathbf{- 1 . 1 4}$ & $2.45 \mathrm{E}-42$ \\
Thy1 & Thy-1/CD90 & -0.69 & $4.05 \mathrm{E}-29$ \\
Fbn1 & Fibrillin-1 & -0.64 & $2.14 \mathrm{E}-33$ \\
Cyp2f2 & Cytochrome P450/Family 2/Subfamily F/Polypeptide 2 & $1.48 \mathrm{E}-12$ \\
Clic5 & Chloride Intracellular Channel Protein 5 & -0.57 & $4.16 \mathrm{E}-38$ \\
\hline
\end{tabular}

bone and cartilage erosions during inflammatory arthritis [3]. Moreover, $M m p 3$ has been shown to be significantly increased in TNF-Tg mice [24] and RA patients [61], which serves as important validation of previously established transcriptional changes in inflammatory arthritis. Prg4 (proteoglycan 4 / lubrican), typically involved in maintaining synovial fluid and tissue homeostasis [62], was also one of the top genes significantly upregulated in TNF-Tg LMCs. While the function of Prg4 expression in PLV-LMCs is unclear, changes in synovial LMCs may mirror these transcriptional modifications, and ought to be investigated in future studies.

To perform a functional genomic analysis on the scRNAseq data, the genes with differential gene expression in TNF-Tg LMCs from Fig. 5A were imported into IPA software, and pathway analysis was performed. A 
graphical summary of the most significantly modified pathways demonstrated downregulation of the pathways that typically inhibit "Organismal death," "Morbidity or mortality," and "Perinatal death," likely leading to the decreased number of TNF-Tg LMCs noted in Fig. 3 (Fig. 5B). Changes in canonical pathways were evaluated where "Oxidative phosphorylation" and "Mitochondrial dysfunction" showed the most significant overlap with differentially expressed genes in TNF-Tg mice, which may exemplify a compensatory response to the ongoing cellular damage (Fig. 5C). The inflammatory diseases associated with the gene expression changes were also identified, and the most significantly related conditions were "Inflammation of joint," "Cell movement of phagocytes," and "Rheumatoid arthritis." This pathway overlap supports the expected inflammatory arthritic phenotype in TNF-Tg mice, and also the increase of inflammatory monocytes determined in Fig. 3 (Fig. 5D). Representative genes Mmp3 (Fig. 5E), Cxcl12 (Fig. 5F), and Ccl19 (Fig. 5G) were significantly increased in TNF-Tg mice, and associated with the pathway modifications noted in TNF-Tg LMCs, all with known involvement in inflammatory arthritis and/or phagocyte chemotaxis [61, 63, 64]. Altogether, these findings both validate and identify the transcriptional changes in TNF-Tg LMCs, which may be related to the progression of arthritis via various mechanisms.

\section{Discussion}

Although lymphatic dysfunction during arthritic progression is well-recognized, the nature of LMCs that are responsible for LV contractions, and how LMCs change during chronic inflammatory arthritis, particularly at the single-cell level, is largely unknown. As new technologies in scRNAseq have enabled many new insights in the arthritis field [65-70], we aimed to use this platform to elucidate the LMC transcriptome and functional genomics involved in joint homeostasis and advanced disease. While the methods for performing scRNAseq at the commencement of this research existed, a major limitation was the means to isolate viable SMCs from PLV-LMCs and SSV-VSMCs. Indeed, this proved to be the most challenging part of our work, as our initial approach focused on recovering large numbers of LMCs following FACS by pooling PLV-LMCs and SSV-VSMCs from several animals ( $n>6$ mice) proved to be ineffective due to the loss of viability of the sorted cells. In the end, we succeeded by harvesting PLV-LMCs and SSV-VSMCs from only three mice at a time, which reduced the ex vivo manipulation time to $<4 \mathrm{~h}$. While this limits the statistical power of individual experiments that can only study approximately 1000-3000 SMCs per scRNAseq, we found replicate scRNAseq experiments to be highly reproducible such that data could be combined in silico to achieve the desired statistical power.

We were also able to utilize fluorescent reporter models of the peripheral SMCs, and through post hoc analysis of the FACS, generate a gating strategy to enrich for SMCs based on the scatter profile of these cells. This approach demonstrated remarkable conservation of the cell types sequenced after fluorescent enrichment for SMCs, and in turn also provided the opportunity for quantitative analysis of crucial peri-vascular immune cell populations that are integral to vascular function between WT and TNF-Tg conditions. However, one of the primary limitations of sorting based solely on scatter is the possibility for batch effect between the experiments as fluorescent markers were not used for specific cell isolation. Given the time constraints previously noted, each scRNAseq experiment was performed on separate days. To reduce the batch effect, especially between the WT and TNF-Tg groups, the exact same sort conditions and gating were performed. The side-by-side comparisons and conservation of cell types when the scRNAseq datasets were integrated in Figs. 2 and 3 suggest limited batch effects in the sequencing process itself.

Here we demonstrated an increase in inflammatory monocytes along with a reduction in LMC and M2-macrophage populations in joint-draining vascular tissues from mice with advanced inflammatory-erosive arthritis. Moreover, TNF-Tg LMCs exhibited significant transcriptomic changes from WT, which were associated with cell death, phagocyte recruitment, joint inflammation, and RA pathogenesis. Together, this study establishes our first understanding of the transcriptional modifications in TNF-Tg LMCs to guide further research on the role of lymphatic contractility in chronic inflammatory arthritis.

The discovery of changes in peri-vascular immune cell populations and reduced LMCs by scRNAseq both corroborates our past work and provides novel hypotheses to further understand the lymphatic pathology in TNF$\mathrm{Tg}$ mice. In fact, recent studies have implicated a direct role for TNF in the apoptosis of LMCs and reduced contractile gene expression, such as Acta2, in synovial lymphatics [39]. Moreover, inflammatory monocytes may be directly recruited to the outside of the vessel by inflamed PLV-LMCs and SSV-VSMCs through increased expression of chemokines, such as Cxcl12, and the accumulation of inflammatory monocytes in the surrounding tissue could reduce lymphatic contractility in TNF-Tg mice through iNOS-mediated mechanisms [15]. The accumulation of these peri-vascular inflammatory monocytes may in part explain the effective restoration of lymphatic function with iNOS inhibition in TNF-Tg mice [16]. Along with increased monocyte recruitment, further study into the permeability of inflamed PLVs in 
TNF-Tg mice is warranted. Collecting lymphatic vessels have been shown to be a point of interaction between permeable lymph contents and surrounding antigenpresenting cells embedded in the adipose tissue that then migrate to draining lymph nodes [71], and may partly explain the dramatic lymph node expansion previously characterized in TNF-Tg mice [9]. The evaluation of perilymphatic immune cells involved in negative regulation of lymphatic contractility and LMC homeostasis in TNF-Tg mice, such as inflammatory monocytes/macrophages and mast cells [72-74], is an active area of investigation. The reduction in M2-macrophages with Lyve1 expression was also previously unappreciated in the TNF-Tg model, and this novel finding is of particular importance given the recently discovered role for peri-vascular Lyve1 expressing M2-macrophages in regulating homeostatic collagen levels in VSMCs, necessary for proper function [38]. In fact, the decrease in M2-macrophages associated with the well-established reduction in TNF-Tg LMC function may be closely related, and ought to be investigated in future studies. Thus, the scRNAseq both validates and provides further understanding of the pathologic mechanisms associated with lymphatic dysfunction in TNF-Tg mice.

However, despite these key findings in our single-cell analysis of the peripheral vasculature, one of the primary limitations of this study is the reliance on scRNAseq to evaluate quantitative and transcriptomic cellular changes. While we were able to identify distinct changes in cell numbers and transcriptomic modifications in TNF-Tg mice, we were unable to elucidate the spatial relationships, cellular interactions, or functional consequences of these changes solely using scRNAseq. For example, although the reduced LMC population in TNF-Tg mice is similar to findings in synovial lymphatics [39], studies of LMC coverage in the collecting PLVs during inflammatory arthritis are still ongoing. In addition, considerable investigation is necessary to determine whether peri-vascular Lyve ${ }^{+}$M2-macrophages localize around PLVs and regulate LMC homeostasis similar to VSMCs [38], and whether the reduction of this cell population is associated with lymphatic pathology in TNF-Tg mice. Future studies are needed to comprehensively evaluate the phenotypic switch towards inflammatory monocytes in these perivascular regions and the impact of these microenvironmental changes on the function of TNF-Tg PLVs. Thus, the current work has generated valuable hypotheses, but will require further validation and investigation in future studies to elucidate the relationship of our findings to the pathology of inflammatory-erosive arthritis.

An unexpected finding of this study was the limited detection of canonical markers for SMCs in the peripheral vasculature of the lower limb. Since RT-PCR approaches have previously demonstrated that LMCs derived from mesenteric and thoracic lymphatic vessels express Acta2 ( $\mathrm{SSMA}$ ) and Myh11 (myosin heavychain 11) [75], we expected these markers to be highly enriched in the SMC populations. However, we further validated the low Acta 2 expression by two different Cre models, Acta2-Cre [20] and Acta2-CreER mice [26], both crossed into the faithful Ai9-tdTomato reporter line [21, 22, 26] with limited tdT expression in PLV-LMCs and SSV-VSMCs.

Moreover, while scRNAseq via droplet-based platforms provides the benefit of high-throughput sequencing, it has been well-established that read depth with this technology is much lower than alternative plate-based approaches, such as Smart-seq2, and thus low frequency transcripts may be dropped in the current study [76]. For example, in a recent study directly comparing $10 \mathrm{x}$ and Smart-seq2 expression patterns by Wang et al. [76], cells derived from a liver tumor were processed and 10x concluded that markers of VSMC contraction in the KEGG pathway were undetectable, while Smart-seq2 identified a significant enrichment of this pathway in the same cell types. Similarly, in a recent study of single-cell profiling of vascular tissue from the brain and lung using Smartseq2, Acta 2 and Myh11 were indeed detectable in venous VSMCs, but at remarkably lower levels compared to the expression of these same genes in arterial VSMCs [77]. Thus, given the relatively low expression of Acta2 and Myh11 in venous VSMCs as measured even with the high read depth of Smart-seq2, the limited expression of canonical SMC genes detected in this study may result from limitations of the low read depth droplet-based sequencing technology. In addition, the zero inflation of certain genes in SMCs may further represent this cellular heterogeneity in the expression of canonical SMC genes across various SMC populations [78]. Given the established diversity and plasticity in SMC phenotypes with a spectrum ranging from expression patterns associated with contractility (i.e., aorta VSMC-like) to synthetic (i.e., fibroblast-like) $[79,80]$, these related cell types may be difficult to differentiate without prior FACS enrichment strategies as SMC and fibroblast cell types may cluster closely together by scRNAseq.

Despite the alignment of tdT-expressing cell populations by whole mount microscopy, flow sorting, and downstream scRNAseq that correspond to the PLVLMCs and SSV-VSMCs, we cannot completely exclude the possibility that proposed LMCs and VSMCs in the current study may represent myofibroblast contaminants from the surrounding tissue. In particular, while noted prototypical SMC genes such as Cald1 and Pdgfrb were highly expressed in the proposed SMC clusters, these genes have also been shown to be expressed by certain 
fibroblast subsets $[77,81]$. As the identification of LMCspecific genes is currently an important goal in the field of lymphatic biology, the potential caveat of myofibroblast contamination must be considered in future investigations. With this in mind, this work does provide enormous promise towards the identification of candidate gene markers that may be used to specifically target LMCs in future studies.

Based on the myofibroblast-like expression pattern of PLV-LMCs and SSV-VSMCs, it remains unclear whether these cells may be directly related to the previously established inflammatory-erosive mechanisms of synovial fibroblasts in RA. The close proximity of the synovial lymphatics to these described fibroblast populations, coupled with the identification of peri-vascular fibroblasts involved in disease pathogenesis [4], raises the question of the relationship between these cell types in inflammatory arthritis that ought to be investigated in future studies. Overall, this study provides further insight on LMC transcriptional changes that drive the wellestablished role of lymphatic dysfunction in inflammatory arthritis, and highlights alterations in peri-vascular immune cell populations. Future studies will focus on further understanding the direct mechanisms between these cellular changes, and the implications on arthritic progression in the afferent joint.

\section{Conclusions}

By scRNAseq, we have identified that chronic inflammatory-erosive arthritis is associated with reduced LMCs and M2-macrophages along with increased inflammatory monocytes in joint-draining vascular tissue. Transcriptional changes in LMCs suggest a direct role in the recruitment of immune cells and pathways known to be involved in joint inflammation, which ought to be investigated in future studies towards a further understanding of the pathogenic mechanisms associated with mesenchymal stromal cells in chronic arthritis.

\footnotetext{
Abbreviations

TNF-Tg: Tumor necrosis factor transgenic; RA: Rheumatoid arthritis; PLVs: Popliteal lymphatic vessels; LMCs: Lymphatic muscle cells; scRNAseq: Singlecell RNA-sequencing; UMAP: Uniform manifold approximation and projection; VSMCs: Vascular smooth muscle cells; PRIME: Preinflammatory mesenchymal; SSV: Superficial saphenous vein; PLN: Popliteal lymph node; LECs: Lymphatic endothelial cells; NO: Nitric oxide; iNOS: Inducible nitric oxide synthase; SMCs: Smooth muscle cells; FACS: Fluorescence activated cell sorting; tdT: tdTomato; WT: Wild-type; DEGs: Differentially expressed genes; BrdU: Bromodeoxyuridine; DMEM: Dulbecco's modified Eagle's medium; FBS: Fetal bovine serum; DPBS: Dulbecco's phosphate buffered saline; FCC: Flow cytometry core; GEM: Gel bead-in-emulsion; SNN: Shared nearest neighbor; Log2FC: $\log _{2}$ fold-change; FDR: False discovery rate; IPA: Ingenuity Pathway Analysis; NBF: Normalized buffered formalin; aSMA: Alpha smooth muscle actin; MPI: Mean pixel intensity; HCT: Micro-computed tomography; HU: Hounsfield units; AU: Artificial units.
}

\section{Supplementary Information}

The online version contains supplementary material available at https://doi. org/10.1186/s13075-022-02730-z.

Additional file 1 : Supplementary Figure 1. Identification of Ptprc ${ }^{+}$ immune and Prrx $1^{+}$mesenchymal clusters by scRNAseq of $\mathrm{tdT}^{+}$sorted cells. A feature plot is shown where Ptprc (CD45) identified immune cell populations (A), and Prrx1 defined the mesenchymal cells containing the smooth muscle cell (SMC) populations and fibroblasts (B). Low quality cell clusters that remained in the dataset were identified by relatively high mitochondrial gene expression indicating dead or dying cells, noted by the arrow $(\mathbf{C})$. Blue $=$ high expression, grey = low expression. Supplementary Figure 2. Differentially expressed genes in cell clusters identified with integration of WT and TNF-Tg datasets. The wild-type (WT) and tumor necrosis factor transgenic (TNF-Tg) single-cell RNA-sequencing (scRNAseq) datasets were integrated together, and unsupervised shared nearest neighbor (SNN) clustering in Seurat resolved 20 distinct cell clusters. The top 3 genes (duplicates omitted) for these 20 cell populations are shown as a heatmap, and the cell populations correspond by color and cluster order. The cell numbers for each population are noted in parentheses, and depict the same populations shown in the UMAPs of Fig. 3. The full gene list defining the cell populations is provided in the Supplementary Materials. Supplementary Figure 3. Micro-CT confirmation of severe inflammatory-erosive arthritis in the experimental TNF-Tg mice. Ex vivo micro-computed tomography ( $\mu \mathrm{CT}$ ) was performed on the ankle joints of the wild-type (WT) (A-B) and tumor necrosis factor transgenic (TNF-Tg) (C-D) mice used in this study. The intact ankle in WT mice (A) shows the expected bone architecture with the talus segmented in blue (B). In contrast, a representative image of a severely eroded ankle in the TNF-Tg mice is depicted (C) with the talus identified in red (D) as an established biomarker of arthritis. The talus bone volumes were quantified in Amira software with a significant decrease in talus volumes in TNF-Tg mice compared to WT (E). An unpaired t-test was used for statistical analysis ( $\left.{ }^{* * *} p<0.0001\right)$. Supplementary Figure 4. Gene expression patterns define various monocyte and macrophage cell populations. Immune cells were first identified by Ptprc (CD45) expression (A), and myeloid cell populations were defined by Itgam (CD11 b) enrichment (B). Patrolling monocytes were defined by $\mathrm{C} \times 3 \mathrm{Cr}$ ( $\mathbf{C}$ ), while inflammatory monocytes were identified by Ly6c2 (D) and C Cr2 (E) expression. M1-polarized macrophages were also enriched in $\mathrm{Ccr} 2$ and $\mathrm{CCl} 2(\mathbf{F})$, while M2-polarized macrophages were identified by MrC1 (G, CD206) and Cd163 (H) expression. Notably, the M2-macrophage population was also enriched for Lyvel (I) known to regulate collagen content in smooth muscle cells [38]. and M2-macrophages were selectively decreased in tumor necrosis factor transgenic (TNF-Tg) mice as noted in Fig. 3. Feature plots overlaying the UMAP are provided to visualize the change in expression (grey $=$ low, blue $=$ high gene expression). Supplementary Figure 5. Additional lymph and angiogenic genes and muscle-related transcription factors selectively expressed in the SMC clusters. Along with the genes indicated in Fig. 4, Vegfa (lymphatic muscle cells (LMCs) 1.10, vascular smooth muscle cells (VSMCs) $1.23 \log _{2}$ fold-change (log2FC) in A), Vegfd (LMCs 1.47, VSMCs $1.00 \log 2 \mathrm{FC}$ in B), and Angpt1 (LMCs 0.29, VSMCs $0.51 \log 2 \mathrm{FC}$ in $\mathbf{C}$ ) as factors critical for lymphangiogenesis and angiogenesis were selectively expressed by LMCs and VSMCs compared to all other cell clusters. In addition, Vegfd known to interact with VEGFR3 to promote lymphangiogenesis [56] demonstrated significantly greater expression in the LMC population compared to VSMCs $(0.47 \log 2 F C$ in $\mathbf{B})$. However, Vegfc expression was nearly undetectable in all cell clusters (D). In addition, muscle-related transcription factors such as Prrx 1 (LMCs 2.22, VSMCs $2.42 \log 2 \mathrm{FC}$ in $\mathbf{E}$ ), Twist1 (LMCs 1.71, VSMCs $1.19 \log 2 \mathrm{FC}$ in $\mathbf{F})$, Tead1 (LMCs 0.64, VSMCs 0.38 log2FC in G), Meox2 (LMCs 0.28, VSMCs 0.94 $\log 2 \mathrm{FC}$ in $\mathbf{H}$ ), Id3 (LMCs 2.37, VSMCs $2.66 \log 2 \mathrm{FC}$ in I), and Csrp2 (LMCs 1.06, VSMCs $1.91 \log 2 \mathrm{FC}$ in $\mathbf{J}$ ) were significantly increased in muscle cell populations compared to all other sequenced cell clusters. While these transcription factors are not individually specific to smooth muscle cells (SMCs), each gene is known to regulate smooth muscle related gene expression and their co-expression in the same cell strongly indicates that the cluster is a muscle cell [47]. Violin plots are shown to demonstrate the effect size between SMC populations compared to all other cell clusters. 
Wilcoxon rank sum test was performed between the different cell types for statistical analysis with false discovery rate (FDR) $<2.87 \mathrm{E}-67$ for all comparisons. Sample sizes: LMCs $=1,985$ cells, VSMCs $=1,331$ cells, Other $=5,563$ cells. Supplementary Figure 6. LMC and VSMC clusters express fibroblast-like genes similar to myofibroblasts, but not pericyte genes. To confirm a lack of contamination by pericytes, canonical pericyte markers such as Cspg4 (A), Mcam (B), and Ngfr (C) were nearly undetectable throughout the dataset. Note that cell sorting of $\mathrm{tdT}^{+}$cells from Cspg4-Cre;Ai9-tdTomato as in Fig. 1 does not presume active Cspg4 expression, but instead that tdTomato $(\mathrm{tdT})^{+}$cells at one time expressed Cspg4. On the other hand, non-specific fibroblast markers were highly enriched in the muscle cell populations, such as Dcn (lymphatic muscle cells (LMCs) 5.25, vascular smooth muscle cells (VSMCs) $5.48 \log _{2}$ fold-change (log2FC) in D), Fbln2 (LMCs 2.12, VSMCs $2.31 \log 2 \mathrm{FC}$ in $\mathbf{E})$, and Pdgfra (LMCs 2.29, VSMCs $2.35 \log 2 \mathrm{FC}$ in F). The various validations of muscle cell identity in Fig. 1, 2, and 4 and Supplementary Figures 5 and 7 suggest that these peripheral muscle cell populations may cluster closely with fibroblasts by single-cell transcriptomics, yet exhibit distinct features to differentiate them from traditional fibroblasts. Violin plots are shown to demonstrate the effect size between muscle cell populations compared to all other cell clusters. Wilcoxon rank sum test was performed between the different cell types for statistical analysis with false discovery rate (FDR) $<2.23 \mathrm{E}-308$ and the lower limit possible in R. Sample sizes: LMCs $=1,985$ cells, VSMCs $=1,331$ cells, Other $=5,563$ cells. Supplementary Figure 7 Differentially expressed genes between LMC and VSMC clusters. The lymphatic muscle cell (LMC) and vascular smooth muscle cell (VSMC) clusters from the wild-type (WT) and tumor necrosis factor transgenic (TNF-Tg) integrated datasets in Fig. 3 were subclustered, and then re-integrated for analysis of differential gene expression between the populations with minimally supervised clustering (A). The top 10 differentially expressed genes between LMCs and VSMCs are shown as a heatmap (B), and the full list of gene expression is provided in the Supplementary Materials. Notable genes, such as Cst3 (2.60 $\log _{2}$ fold-change $(\log 2 \mathrm{FC})$ in $\mathbf{C}), \mathrm{Mgp}(2.75 \log 2 \mathrm{FC}$ in D), and Bgn (2.11 $\log 2 \mathrm{FC}$ in $\mathbf{E}$ ), were significantly increased in VSMCs compared to LMCs, while Ackr3 (1.39 $\log 2 \mathrm{FC}$ in $\mathbf{F}$ ) was selective for LMCs relative to VSMCs. Feature plots overlaying the UMAP are provided to visualize the change in expression (grey $=$ low, blue $=$ high gene expression), while violin plots are shown to demonstrate the effect size between muscle cell populations. Wilcoxon rank sum test was performed between the different cell types for statistical analysis with false discovery rate (FDR) $<2.53 \mathrm{E}-227$ for all comparisons. Sample sizes: LMCs $=1,850$ cells, VSMCs $=1,466$ cells. Supplementary Figure 8. Lack of Acta2 expression in LMC and VSMC clusters. Acta2 (alpha smooth muscle actin (aSMA)) is a well-established canonical marker for smooth muscle cells (SMCs), and immunohistochemistry for aSMA has been routinely used by us and others to study lymphatic muscle cells (LMCs) and vascular smooth muscle cells (VSMCs) in lymphatic and blood vessels in situ and ex vivo (Fig. 4 and [22, 43, 75, 82]). Transcriptomic studies of large arteries have also used Acta2 expression as a phenotypic marker of SMC clusters in single-cell RNA-sequencing (scRNAseq) datasets [80, 83]. However, our scRNAseq data showed that Acta2 expression is nearly undetectable in freshly harvested and sequenced popliteal lymphatic vessel (PLV)-LMCs and superficial saphenous vein (SSV)-VSMCs (A, B). A feature plot overlaying the UMAP is provided to visualize the change in expression (grey $=$ low, blue $=$ high gene expression), while a violin plot is shown to demonstrate the negligible Acta2 expression in all sequenced cell populations. We also generated Acta2-Cre;Ai9-tdTomato (tdT) (C-D) and Acta2-CreER;Ai9-tdT (E-J) reporter mice to evaluate endogenous Acta2 gene promoter activity in LMCs and VSMCs in vivo. PLVs (dashed arrow) and adjacent SSVs (solid arrow) from a 3-month-old Acta2-Cre;Ai9-tdT mouse, and a 5-month-old Acta2-CreER;Ai9-tdT mouse (after tamoxifen induction, as previously described [22]) were harvested and immunostained for aSMA and visualized by whole mount immunofluorescent microscopy. High-magnification images of the regions with the highest tdT expression are shown. Remarkably, despite LMC and VSMC aSMA protein expression detected by immunostaining (green), tdT (red) in both reporter models was not ubiquitously expressed in SMCs of both PLVs and SSVs. Taken together, these findings suggest relatively low Acta2 mRNA expression in LMCs and VSMCs of lower limb vessels in adult mice.

\section{Additional file 2.}

\section{Acknowledgements}

We would like to thank the staff and faculty of the following cores and centers at the University of Rochester for their support and efforts throughout this project: (1) Flow Cytometry Core, (2) Genomics Research Center, (3) Center for Advanced Light Microscopy and Nanoscopy, (4) Histology Biochemistry, and Molecular Imaging Core, and (5) Biomechanics and Multimodal Tissue Imaging Core. We would also like to thank Dr. Ivo Kaljzic at the University of Connecticut for sharing the Acta2-CreER mice used in this study.

\section{Authors' contributions}

HMK and EMS contributed to the conception and design of the work. All authors contributed to the acquisition, analysis, and interpretation of the data along with drafting and revising the work. All author(s) read and approved the final manuscript.

\section{Funding}

T32GM007356, T32AR076950 (HMK), R00AR075899 \& OREF (CLW), R01AR073169 (AEL), R01AR063650, R01AG059775 (LX), R01AR069000 (CTR), R01AR056702 (EMS), and P30 AR069655.

\section{Availability of data and materials}

The scRNAseq datasets generated in the current study are deposited on $\mathrm{NIH}$ Gene Expression Omnibus (GEO; accession number GSE190999), and source code will be made available upon reasonable request. All other datasets generated or analyzed during this study are either included in this published article (and its supplementary information files) or will be made available upon reasonable request.

\section{Declarations}

\section{Ethics approval and consent to participate}

All animal studies were approved by the University Committee on Animal Resources (UCAR) at the University of Rochester, and experiments were performed within an Association for Assessment and Accreditation of Laboratory Animal Care (AAALAC)-accredited vivarium.

\section{Consent for publication \\ Not applicable.}

\section{Competing interests}

The authors declare that they have no competing interests.

\section{Author details}

${ }^{1}$ Center for Musculoskeletal Research, University of Rochester Medical Center, 601 Elmwood Ave, Box 665, Rochester, NY 14642, USA. ² Department of Pathology \& Laboratory Medicine, University of Rochester Medical Center, Rochester, NY, USA. ${ }^{3}$ Department of Orthopaedics, University of Rochester Medical Center, Rochester, NY, USA. ${ }^{4}$ Department of Medicine, Division of Allergy, Immunology, Rheumatology, University of Rochester Medical Center, Rochester, NY, USA.

Received: 13 October 2021 Accepted: 21 January 2022

Published online: 07 March 2022

\section{References}

1. Firestein $\mathrm{G}$. The disease formerly known as rheumatoid arthritis. Arthritis Res Ther. 2014;16:114

2. Lefevre S, Meier F, Neumann E, Muller-Ladner U. Role of synovial fibroblasts in rheumatoid arthritis. Curr Pharm Des. 2015;21(2):130.

3. Croft A, Campos J, Jansen K, Turner J, Marshall J, Attar M, et al. Distinct fibroblast subsets drive inflammation and damage in arthritis. Nature. 2019;570(7760):246.

4. Mizoguchi F, Slowikowski K, Wei K, Marshall J, Rao D, Chang S, et al. Functionally distinct disease-associated fibroblast subsets in rheumatoid 
arthritis. Nature. Communications. 2018;9(789). https://doi.org/10.1038/ s41467-018-02892-y.

5. Orange D, Yao V, Sawicka K, Fak J, Frank M, Parveen S, et al. RNA Identification of PRIME cells predicting rheumatoid arthritis flares. N Engl J Med. 2020;383:218.

6. Bouta E, Bell R, Rahimi H, Xing L, Wood R, Bingham C 3rd, et al. Targeting lymphatic function as a novel therapeutic intervention for rheumatoid arthritis. Nat Rev Rheumatol. 2018;14(2):94.

7. Von der Weid P, Zawieja D. Lymphatic smooth muscle: the motor unit of lymph drainage. Int J Biochem Cell Biol. 2004;36(7):1147.

8. Bouta E, Li J, Ju Y, Brown E, Ritchlin C, Xing L, et al. The role of the lymphatic system in inflammatory-erosive arthritis. Semin Cell Dev Biol. 2015;38:90

9. Li J, Kuzin I, Moshkani S, Proulx S, Xing L, Skrombolas D, et al. Expanded $\mathrm{CD} 23(+) / C D 21$ (hi) B cells in inflamed lymph nodes are associated with the onset of inflammatory-erosive arthritis in tnf-transgenic mice and are targets of anti-CD20 therapy. J Immunol. 2010;184(11):6142.

10. Bouta E, Ju Y, Rahimi H, De Mesy BK, Wood R, Xing L, et al. Power Doppler ultrasound phenotyping of expanding versus collapsed popliteal lymph nodes in murine inflammatory arthritis. PLoS One. 2013;8(9):e73766.

11. Bouta E, Kuzin I, De Mesy BK, Wood R, Rahimi H, Ji R, et al. Brief report: treatment of tumor necrosis factor-transgenic mice with anti-tumor necrosis factor restores lymphatic contractions, repairs lymphatic vessels, and may increase monocyte/macrophage egress. Arthritis Rheum. 2017;69(6):1187.

12. Li J, Zhou Q, Wood R, Kuzin I, Bottaro A, Ritchlin C, et al. CD23+/CD21 hi $B$ cell translocation and ipsilateral lymph node collapse is associated with asymmetric arthritic flare in TNF-Tg mice. Arthritis Res Ther. 2011;13(4):R138.

13. Scallan J, Bouta E, Rahimi H, Kenney H, Ritchlin C, Davis M, et al. Ex vivo demonstration of functional deficiencies in popliteal lymphatic vessels from TNF-Tg mice with inflammatory arthritis. Front. Physiology. 2021;12(745096). https://doi.org/10.3389/fphys.2021.745096.

14. Bell R, Rahimi H, Kenney H, Lieberman A, Wood R, Schwarz E, et al. Altered lymphatic vessel anatomy and markedly diminished lymphatic clearance in the rheumatoid hand with active arthritis. Arthritis Rheum. 2020;72(9):1447.

15. Liao S, Cheng G, Conner D, Huang Y, Kucherlapati R, Munn L, et al. Impaired lymphatic contraction associated with immunosuppression. Proc Natl Acad Sci U S A. 2011;108(46):18784

16. Liang Q, Ju Y, Chen Y, Wang W, Li J, Zhang L, et al. Lymphatic endothelial cells efferent to inflamed joints produce iNOS and inhibit lymphatic vessel contraction and drainage in TNF-induced arthritis in mice. Arthritis Res Ther. 2016;18(62). https://doi.org/10.1186/s13075-016-0963-8.

17. Li J, Ju Y, Bouta E, Xing L, Wood R, Kuzin I, et al. Efficacy of B cell depletion therapy for murine joint arthritis flare is associated with increased lymphatic flow. Arthritis Rheum. 2013;65(1):130.

18. Bell R, Slattery P, Wu E, Xing L, Ritchlin C, Schwarz E. iNOS dependent and independent phases of lymph node expansion in mice with TNF-induced inflammatory-erosive arthritis. Arthritis Res Ther. 2019;21:240.

19. Bell R, Wu E, Rudmann C, Forney M, Kaiser C, Wood R, et al. Selective sexual dimorphisms in musculoskeletal and cardiopulmonary pathologic manifestations and mortality incidence in the tumor necrosis factortransgenic mouse model of rheumatoid arthritis. Arthritis Rheum. 2019:71(9):1512

20. LeBleu V, Taduri G, O'Connell J, Teng Y, Cooke V, Woda C, et al. Origin and function of myofibroblasts in kidney fibrosis. Nat Med. 2013;19(8):1047.

21. Madisen L, Zwingman T, Sunkin S, Oh S, Zariwala H, Gu H, et al. A robust and high-throughput Cre reporting and characterization system for the whole mouse brain. Nat Neurosci. 2010;13(1):133.

22. Kenney H, Bell R, Masters E, Xing L, Ritchlin C, Schwarz E. Lineage tracing reveals evidence of a popliteal lymphatic muscle progenitor cell that is distinct from skeletal and vascular muscle progenitors. Sci Rep. 2020;10(18088). https://doi.org/10.1038/s41598-020-75190-7.

23. Douni E, Akassoglou K, Alexopoulou L, Georgopoulos S, Haralambous S, Hill S, et al. Transgenic and knockout analyses of the role of TNF in immune regulation and disease pathogenesis. J Inflamm. 1995-1996;47(1-2):27.

24. Li P, Schwarz E. The TNF-alpha transgenic mouse model of inflammatory arthritis. Springer Semin Immunopathol. 2003;25(1):19.
25. Cuervo H, Pereira B, Nadeem T, Lin M, Lee F, Kitajewski J, et al. PDGFRßP2A-CreER T2 mice: a genetic tool to target pericytes in angiogenesis. Angiogenesis. 2017;20(4):655.

26. Grcevic D, Pejda S, Matthews B, Repic D, Wang L, Li H, et al. In vivo fate mapping identifies mesenchymal progenitor cells. Stem Cells. 2011;30(2):187.

27. Butler A, Hoffman P, Smibert P, Papalexi E, Satija R. Integrating singlecell transcriptomic data across different conditions, technologies, and species. Nat Biotechnol. 2018:36:411.

28. Hao Y, Hao S, Andersen-Nissen E, Mauck W III, Zheng S, Butler A, et al. Integrated analysis of multimodal single-cell data. Cell. 2021;184(13):3573.

29. Satija R, Farrell J, Gennert D, Schier A, Regev A. Spatial reconstruction of single-cell gene expression data. Nat Biotechnol. 2015;33:495.

30. Stuart T, Butler A, Hoffman P, Hafemeister C, Papalexi E, Mauck W III, et al. Comprehensive Integration of Single-Cell Data. Cell. 2019;177(7):1888

31. McInnes L, Healy J, Melville J. UMAP: Uniform Manifold Approximation and Projection for Dimension Reduction. arXiv. 2018;1802.03426.

32. Wickham H. ggplot2: Elegant Graphics for Data Analysis; 2016.

33. Proulx S, Kwok E, You Z, Papuga M, Beck C, Shealy D, et al. Longitudinal assessment of synovial, lymph node, and bone volumes in inflammatory arthritis in mice by in vivo magnetic resonance imaging and microfocal computed tomography. Arthritis Rheum. 2007:56(12):4024.

34. Kapellos T, Bonaguro L, Gemünd I, Reusch N, Saglam A, Hinkley E, et al. Human monocyte subsets and phenotypes in major chronic inflammatory diseases. Front Immunol. 2019;10(2035). https://doi.org/10.3389/ fimmu.2019.02035.

35. Ochocka N, Segit P, Walentynowicz K, Wojnicki K, Cyranowski S, Swatler $J$, et al. Single-cell RNA sequencing reveals functional heterogeneity of glioma-associated brain macrophages. Nature. Communications. 2021;12(1151). https://doi.org/10.1038/s41467-021-21407-w.

36. Ambarus C, Krausz S, Eijk M, Hamann J, Radstake T, Reedquist K, et al. Systematic validation of specific phenotypic markers for in vitro polarized human macrophages. J Immunol Methods. 2012;375(1-2):196.

37. Carson W IV, Salter-Green S, Scola M, Joshi A, Gallagher K, Kunkel S. Enhancement of macrophage inflammatory responses by CCL2 is correlated with increased miR-9 expression and downregulation of the ERK1/2 phosphatase Dusp6. Cell Immunol. 2018;314:63.

38. Lim H, Lim S, Tan C, Thiam C, Goh C, Carbajo D, et al. Hyaluronan receptor LYVE-1-expressing macrophages maintain arterial tone through hyaluronan-mediated regulation of smooth muscle cell collagen. Immunity. 2018:49(2):326

39. Liang Q, Zhang L, Xu H, Li J, Chen Y, Schwarz E, et al. Lymphatic muscle cells contribute to dysfunction of the synovial lymphatic system in inflammatory arthritis in mice. Arthritis Res Ther. 2021;23(58). https://doi. org/10.1186/s13075-021-02438-6.

40. Marston S, Burton D, Copeland O, Fraser I, Gao Y, Hodgkinson J, et al. Structural interactions between actin, tropomyosin, caldesmon and calcium binding protein and the regulation of smooth muscle thin filaments. Acta Physiol Scand. 1998;164(4):401.

41. Gallant C, Appel S, Graceffa P, Leavis P, Lin JJC, Gunning P, et al. Tropomyosin variants describe distinct functional subcellular domains in differentiated vascular smooth muscle cells. Am J Physiol Cell Physiol. 2011;300(6):C1356.

42. Owens $\mathrm{G}$. Regulation of differentiation of vascular smooth muscle cells. Physiol Rev. 1995;75(3):487.

43. Wang $Y$, Jin $Y$, Mäe $M$, Zhang $Y$, Ortsäter $H$, Betsholtz $C$, et al. Smooth muscle cell recruitment to lymphatic vessels requires PDGFB and impacts vessel size but not identity. Development. 2017;144(19):3590.

44. Hellström M, Kalen M, Lindahl P, Abramsson A, Betsholtz C. Role of PDGF-B and PDGFR-beta in recruitment of vascular smooth muscle cells and pericytes during embryonic blood vessel formation in the mouse. Development. 1999;126(14):3047.

45. Ferrara N, Winer J, Burton T. Aortic smooth muscle cells express and secrete vascular endothelial growth factor. Growth Factors. 1991;5(2):141.

46. Holmes D, Zachary I. The vascular endothelial growth factor (VEGF) family: angiogenic factors in health and disease. Genome Biol. 2005;6(2):209.

47. Kumar M, Owens G. Combinatorial control of smooth muscle-specific gene expression. Arterioscler Thromb Vasc Biol. 2003;23(5):737. 
48. Kumar A, D'Souza S, Moskvin O, Toh H, Wang B, Zhang J, et al. Specification and diversification of pericytes and smooth muscle cells from mesenchymoangioblasts. Cell Rep. 2017;19(9):1902.

49. Guerrero-Juarez C, Dedhia P, Suogin J, Ruiz-Vega R, Ma D, Liu Y, et al. Single-cell analysis reveals fibroblast heterogeneity and myeloidderived adipocyte progenitors in murine skin wounds. Nat Commun. 2019;10:650.

50. Shi G, Sukhova G, Grubb A, Ducharme A, Rhode L, Lee R, et al. Cystatin C deficiency in human atherosclerosis and aortic aneurysms. J Clin Invest. 1999;104(9):1191.

51. Luo G, Ducy P, McKee M, Pinero G, Loyer E, Behringer R, et al. Spontaneous calcification of arteries and cartilage in mice lacking matrix GLA protein. Nature. 1997;386(6620):78.

52. Asokan P, Mitra R, Periasamy R, Han Z, Borras T. A naturally fluorescent Mgp transgenic mouse for angiogenesis and glaucoma longitudinal studies. Invest Ophthalmol Vis Sci. 2018;59(2):746.

53. Shimizu-Hirota R, Sasamura H, Kuroda M, Kobayashi E, Hayashi M, Saruta T. Extracellular matrix glycoprotein biglycan enhances vascular smooth muscle cell proliferation and migration. Circ Res. 2004;94(8):1067.

54. Nikkari S, Järveläinen $\mathrm{H}$, Wight $\mathrm{T}$, Ferguson $\mathrm{M}$, Clowes A. Smooth muscle cell expression of extracellular matrix genes after arterial injury. Am J Pathol. 1994;144(6):1348.

55. Sigmund E, Baur L, Schineis P, Arasa J, Collado-Diaz V, Vranova M, et al. Lymphatic endothelial-cell expressed ACKR3 is dispensable for postnatal lymphangiogenesis and lymphatic drainage function in mice. PLoS One. 2021;16(4):e0249068.

56. Achen $M$, Jeltsch $M$, Kukk E, Mäkinen T, Vitali A, Wilks A, et al. Vascular endothelial growth factor D (VEGF-D) is a ligand for the tyrosine kinases VEGF receptor 2 (Flk1) and VEGF receptor 3 (Flt4). Proc Natl Acad Sci U S A 1998;95(2):548.

57. Baldwin M, Catimel B, Nice E, Roufail S, Hall N, Stenvers K, et al. The specificity of receptor binding by vascular endothelial growth factor-d is different in mouse and man. J Biol Chem. 2001;276(22):19166.

58. Chakraborty R, Saddouk F, Carrao A, Krause D, Greif D, Martin K. Promoters to study vascular smooth muscle. Arterioscler Thromb Vasc Biol. 2019;39(4):603.

59. Kim F, Barnes E, Ying L, Chen C, Lee L, Alvira C, et al. Pulmonary artery smooth muscle cell endothelin-1 expression modulates the pulmonary vascular response to chronic hypoxia. Am J Physiol Lung Cell Mol Physiol. 2015;308(4):L368

60. Garcia-Alvarez O, Fisch K, Wineinger N, Akagi R, Saito M, Sasho T, et al. Increased DNA methylation and reduced expression of transcription factors in human osteoarthritis cartilage. Arthritis Rheumatol. 2016;68(8):1876.

61. Zucker S, Hymowitz M, Conner C, Zarrabi H, Hurewitz A, Matrisian L, et al. Measurement of matrix metalloproteinases and tissue inhibitors of metalloproteinases in blood and tissues. Clinical and experimental applications. Ann N Y Acad Sci. 1999;878:212.

62. Rhee D, Marcelino J, Baker M, Gong Y, Smits P, Lefebvre V, et al. The secreted glycoprotein lubricin protects cartilage surfaces and inhibits synovial cell overgrowth. J Clin Invest. 2005;115(3). https://doi.org/10. $1172 / \mathrm{JCl} 200522263$

63. Pickens S, Chamberlain N, Volin M, Pope R, Mandelin A II, Shahrara S Characterization of CCL19 and CCL21 in rheumatoid arthritis. Arthritis Rheumatol. 2011;63(4):914.

64. Klerck B, Geboes L, Hatse S, Kelchtermans H, Meyvis Y, Vermeire K, et al. Pro-inflammatory properties of stromal cell-derived factor-1 (CXCL12) in collagen-induced arthritis. Arthritis Res Ther. 2005;7(6):R1208.

65. Alivernini S, MacDonald L, Elmesmari A, Finlay S, Tolusso B, Gigante MR, et al. Distinct synovial tissue macrophage subsets regulate inflammation and remission in rheumatoid arthritis. Nat Med. 2020;26(8):1295-306.

66. Buckley CD. Macrophages form a protective cellular barrier in joints. Nature. 2019;572(7771):590-2

67. Wei K, Korsunsky I, Marshall JL, Gao A, Watts GFM, Major T, et al. Notch signalling drives synovial fibroblast identity and arthritis pathology. Nature. 2020:582(7811):259-64.

68. Zhang F, Wei K, Slowikowski K, Fonseka C, Rao D, Kelly S, et al. Defining inflammatory cell states in rheumatoid arthritis joint synovial tissues by integrating single-cell transcriptomics and mass cytometry. Nat Immunol. 2019;20(7):928
69. Der E, Suryawanshi H, Morozov P, Kustagi M, Goilav B, Ranabothu S, et al. Tubular cell and keratinocyte single-cell transcriptomics applied to lupus nephritis reveal type I IFN and fibrosis relevant pathways. Nat Immunol. 2019;20(7):915.

70. Zhang F, Mears J, Shakib L, Beynor J, Shanaj S, Korsunsky I, et al. IFN-y and TNF-a drive a CXCL10+ CCL2+ macrophage phenotype expanded in severe COVID-19 lungs and inflammatory diseases with tissue inflammation. Genome Med. 2021;13(64). https://doi.org/10.1186/ s13073-021-00881-3.

71. Kuan E, Ivanov S, Bridenbaugh E, Victora G, Wang W, Childs E, et al. Collecting lymphatic vessel permeability facilitates adipose tissue inflammation and distribution of antigen to lymph node-homing adipose tissue dendritic cells. J Immunol. 2015;194(11):5200.

72. Shang T, Liang J, Kapron C, Liu J. Pathophysiology of aged lymphatic vessels. Aging. 2019;11(16):6602.

73. Pal S, Nath S, Meininger C, Gashev A. Emerging roles of mast cells in the regulation of lymphatic immuno-physiology. Front Immunol. 2020;11(1234). https://doi.org/10.3389/fimmu.2020.01234.

74. Scallan J, Davis M. Itching for answers: how histamine relaxes lymphatic vessels. Microcirculation. 2014:21(7):575

75. Muthuchamy M, Gashev A, Boswell N, Dawson N, Zawieja D. Molecular and functional analyses of the contractile apparatus in lymphatic muscle. FASEB J. 2003;17(8):920.

76. Wang X, He Y, Zhang Q, Ren X, Zhang Z. Direct comparative analyses of 10X genomics chromium and Smart-seq2. Genomics Proteomics Bioinformatics. 2021;19(2):253.

77. He L, Vanlandewijck M, Mäe M, Andrae J, Ando K, Gaudio F, et al. Singlecell RNA sequencing of mouse brain and lung vascular and vessel-associated cell types. Sci Data. 2018;5(180160). https://doi.org/10.1038/sdata. 2018.160.

78. Choi K, Chen Y, Skelly D, Churchill G. Bayesian model selection reveals biological origins of zero inflation in single-cell transcriptomics. Genome Biol. 2020;21(183). https://doi.org/10.1186/s13059-020-02103-2.

79. Shanahan C, Weissberg P. Smooth muscle cell heterogeneity: patterns of gene expression in vascular smooth muscle cells in vitro and in vivo. Arterioscler Thromb Vasc Biol. 1998;18(3):333.

80. Dobnikar L, Taylor A, Chappell J, Oldach P, Harman J, Oerton E, et al. Disease-relevant transcriptional signatures identified in individual smooth muscle cells from healthy mouse vessels. Nature Commun. 2018;9(4567). https://doi.org/10.1038/s41467-018-06891-x.

81. Vanlandewijck M, He L, Mäe M, Andrae J, Ando K, Gaudio F, et al. A molecular atlas of cell types and zonation in the brain vasculature. Nature. 2018;554:475.

82. Jones D, Meijer E, Blatter C, Liao S, Pereira E, Bouta E, et al. Methicillinresistant Staphylococcus aureus causes sustained collecting lymphatic vessel dysfunction. Sci Transl Med. 2018;10(424):eaam7964.

83. Li Y, Ren P, Dawson A, Vasquez H, Ageedi W, Zhang C, et al. Single-cell transcriptome analysis reveals dynamic cell populations and differential gene expression patterns in control and aneurysmal human aortic tissue. Circulation. 2020;142(14):1374.

\section{Publisher's Note}

Springer Nature remains neutral with regard to jurisdictional claims in published maps and institutional affiliations. 